Training a New Instrument to Measure Cotton Fiber Maturity

Using Transfer Learning

by

Christopher N. Turner, B.S., M.S.

A Dissertation

In

\title{
ELECTRICAL AND COMPUTER ENGINEERING
}

Submitted to the Graduate Faculty

of Texas Tech University in

Partial Fulfillment of

the Requirements for

the Degree of

DOCTOR OF PHILOSPHY

Pending Approval

Dr. Hamed Sari-Sarraf

Chair of Committee

Dr. Eric Hequet

Dr. Ranadip Pal

Dr. Mark Sheridan

Dean of the Graduate School

December 2016 
Copyright 2016, Christopher N. Turner 


\section{ACKNOWLEDGMENTS}

This work would not have been possible without a great deal of help. First and foremost, it has been a privilege working under the direction of my committee chair, Dr. Hamed Sari-Sarraf, as well as Dr. Eric Hequet for the past several years. I thank both for their guidance and patience with me, as well as the opportunities and support they have provided. I have been very fortunate to have this opportunity to work with both of them. I would like to thank Dr. Ranadip Pal for serving on my committee and providing thoughtful suggestions despite such short notice. I would also like to thank Raquel Ruiz at the TTU Fiber and Biopolymer Institute for her dedication and patience during the countless hours of scanning thousands of individual cotton fibers. My family and I would like to express gratitude to Cotton Inc. for their financial support over the years. Along those lines, I am also very thankful for Mike Crowder at the TTU Health Sciences Center for allowing me to work around my school schedule including switching between part-time and full-time employment when needed.

Last, but certainly not least, I would like to thank my family and friends for their support and for putting up with and without me over the past few yearsparticularly in recent months. I am especially thankful for my amazing wife, Kim, for her patience and support at home while I pursued this final degree. 


\section{TABLE OF CONTENTS}

ACKNOWLEDGMENTS .............................................................................. ii

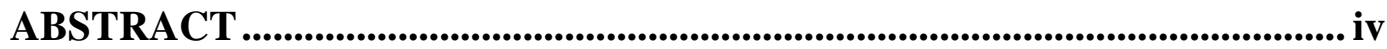

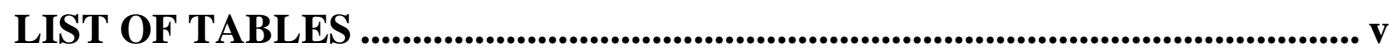

LIST OF FIGURES .................................................................................................... vi

CHAPTER I INTRODUCTION_.................................................................. 1

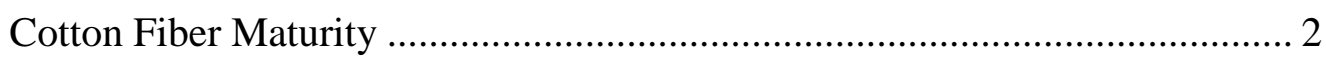

Transfer Learning — Prior Work............................................................. 5

CHAPTER II METHODS......................................................................................... 8

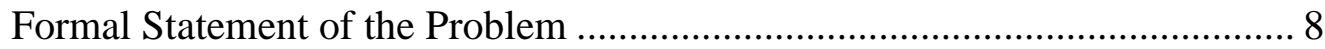

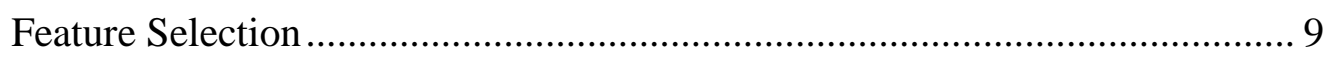

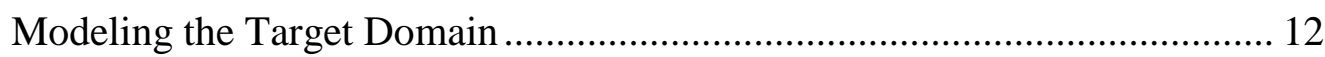

Modeling Algorithm Details ......................................................................... 15

CHAPTER III RESULTS ......................................................................................... 20

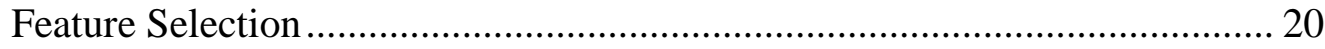

Modeling the Target Domain ........................................................................ 23

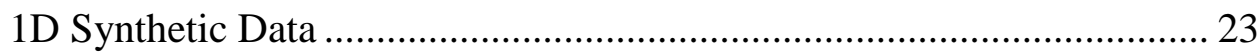

2D Synthetic Data ................................................................................... 27

Real World Data.................................................................................. 30

CHAPTER IV ESTIMATING COTTON FIBER MATURITY ....................... 35

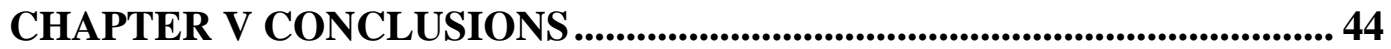

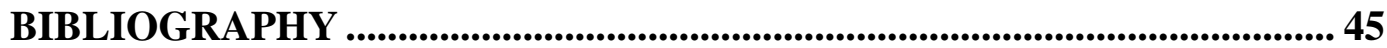




\begin{abstract}
This dissertation presents novel transfer learning feature selection and regression methods that utilizes data from an older instrument to train a new instrument to assess the same measurement. The method assumes that the instruments measure the same property but by different methodologies, and that samples presented to one apparatus are not available to the other. The algorithm makes use of a single feature common to both instruments to create a link with which to transfer information regarding the distribution of the resulting measurements, or labels. The goal is to generate a model in the domain of the new instrument that maps data from analyzed samples to an output measurement. This modeling process is accomplished through an iterative algorithm that supports many types of regression schemes. Results are shown using both synthetic and real world data sets, which demonstrate the effectiveness of the proposed method. Finally, we present how this technique is used to train a new instrument designed to measure cotton fiber maturity.
\end{abstract}




\section{LIST OF TABLES}

3.1 Means of the Six Categories in the Source and Target Domains.................... 21

3.2 Feature Selection - Synthetic Data Results .................................................. 23

3.4 Adding Bias to the Target Distributions ........................................................ 30

3.5 Model Selection - Cross Section Data as Target Domain ............................... 33

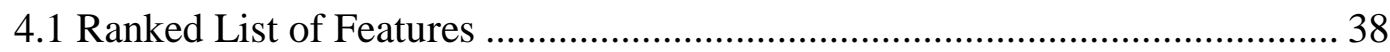

4.2 Model Selection - Vision-Based Maturity measurement .................................. 39 


\section{LIST OF FIGURES}

Fig. 1.1 Diagram illustrating the task at hand. The goal is to train a new measurement system using data from an old measurement system via transfer learning.

Fig. 1.2. (a) Diagram of the cross section of a cotton fiber. (b) Microscopic image of cotton fiber cross sections. (c) Image segment of a cotton fiber from the system described by [8].

Fig. 2.1. Simple 1D example of a neighborhood where (2.6) does not hold

Fig. 3.1. Synthetic data used to test the feature selection algorithm. (a)

Two source domain features. (b) Three good target domain features.

Fig. 3.2. An example of the behavior of the criterion function (2.2).

Each line is an example of one run of the algorithm taken from each batch in the second row of Table 2 where $\sigma \mathrm{S}=1$.

Fig. 3.3. Demonstration of modeling algorithm on 1D synthetic data with a quadratic model $y=3 \times 2$. (a) Synthetic data of three categories with labels assigned by the true model.

(b) Initialized labels according to (2.4). (c) Result of first modeling attempt. First iteration: (d) labels are reassigned via histogram matching and (e) modeling result after histogram matching. Second iteration: (f) histogram matching and (g) modeling. (h) Plot of M (Eq. (2.9)) over the five iterations. (i) R2 (Eq. (2.10)) over five iterations.

Fig. 3.4. An illustration of the scenario when (2.6) does not hold (shaded regions). (a) Labels are assigned according to a simple linear model. (b) Histogram matching is applied.

Fig. 3.5. A 2D example of synthetic data. (a) Source domain with the shade of the point relating to label value. (b) Target domain with true labels. (c) Target domain with initialized labels. (d) Plot of predicted versus true labels after completion of the algorithm.

Fig. 3.6. Distribution of maturity for the five bales chosen as training data. The bale numbers in the legend (3212, 3042, etc.) correspond to specific bales discussed by Hequet et al. 
Fig. 3.7. Cotton fiber cross section data. (a) Target data with true labels.

(b) Target data with initialized labels.

Fig. 3.8. Scatter plot of predicted labels versus true labels for each of the models attempted using the cotton fiber cross section data as the target. (a) Linear. (b) Quadratic. (c) Logistic

Fig. 4.1. Contour plots of density estimates of samples from the target domain for two particular features, i.e. $\mathrm{P}(\mathrm{xT} \mid \mathrm{cj} \mathrm{T})$ : shape factor and homogeneity. Graphs are arranged from highest maturity to lowest maturity according to the distributions in Fig. 3.6: (a) Bale 3212, (b) Bale 3042, (c) Bale 3029, (d) Bale 3057, and (e) Bale 3116.

Fig. 4.2. (a) Results of running the feature selection algorithm on the line scan data set. Each point shows the value of the criterion function as each feature is removed. The minimum occurs when the eleventh feature is removed. (b) Results of applying five different models to subsets of the top seven features. 38

Fig. 4.3. (a)-(e) Density estimate of maturity for each of the five cotton bales in the cross section domain (source) and the predicted maturity based on the "Logistic-Quadratic" model for the vision-based measurement (target). (f) Distribution of predicted maturity for each bale in the target domain. Compare to Fig. 3.6.

Fig. 4.4. Results of samples taken from three bales used to test the chosen "Logistic-Quadratic" model. Each graph shows the source label distributions as well as the predicted target label distributions. (a) Bale 3129 (b) Bale 3056 (c) Bale 3140 42

Fig. 4.5. Twelve of the most (a) mature and (b) immature fiber segments from Bale 3056 using predicted values based on the "Logistic-Quadratic" model. Predicted maturity values appear above each segment 


\section{CHAPTER I INTRODUCTION}

The development of new instruments is often pursued with the goal of replacing existing measurement methods to increase accuracy, efficiency, or convenience. In many cases, the new instrument may provide the same measurement in a completely different manner. For example, in [1], a vision-based method is presented to measure yarn density in woven fabrics, which replaces an older method using X-ray sensors. In [2], a new sensor is designed to measure fabric texture in a manner that mimics human touch. This new instrument is proposed to replace older methods based on microscopic examination or more recent techniques that make use of computer vision. In each of these new systems, calibration and validation of the new method is achieved by measuring the same object in both systems. Even with nondestructive methods which replace destructive techniques, in many cases an object can first be measured by the nondestructive method and then by the destructive method for comparison, such as in [3].

However, in this paper, we present a scenario in which the measurement methods either destroy their respective samples (e.g. blood tests such as those described by [4]) or differ in the manner by which they accept the samples (e.g. one in batch form, the other by individual samples as is the case with many different cotton fiber quality measurements [5]). These conditions prohibit a direct comparison between old and new techniques for an individual specimen. In order to overcome this problem, we propose a new method based on transfer learning that allows a new instrument to learn how to evaluate the same measurement as an older instrument. We assume that these two instruments measure the same property but by different methodologies, and that samples presented to one apparatus are not available to the other. As illustrated by Fig. 1.1, the idea is to use existing measurement data from the old instrument as a means to transfer knowledge to the new instrument. Although, we 
present an application of this new method in the measurement of cotton fiber maturity, we believe the technique is applicable to a general class of similar problems.

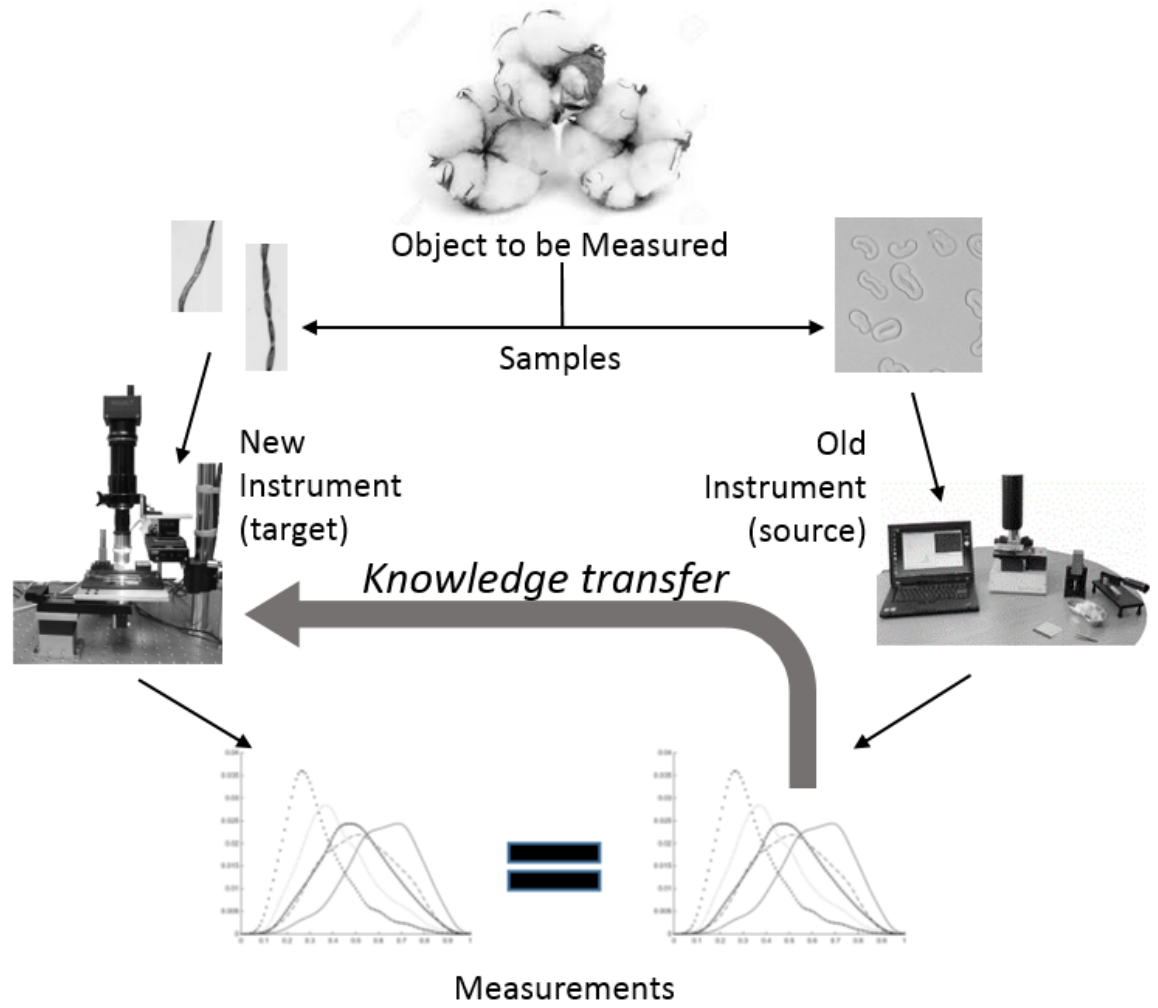

Fig. 1.1 Diagram illustrating the task at hand. The goal is to train a new measurement system using data from an old measurement system via transfer learning.

\section{Cotton Fiber Maturity}

One of the most important characteristics used to evaluate cotton fiber quality is maturity. A single cotton fiber exhibits a tube-like structure. As the fiber matures, the inside of the tube is filled with cellulose. This measurement describes the amount of cellulose that is deposited within a fiber relative to the circumference of its cross section. More mature fibers have better dye affinity and are stronger, which allows them to survive manufacturing processes without breaking; thus, producing better quality yarns. 
The current, most reliable, and direct method calculates maturity by analyzing microscopic images of fiber cross-sections [6]. Using this method, bundles of fibers, prepared in specific manner prescribed by the standard [6], must be cut by a microtome into $1 \mu \mathrm{m}$-thick cross sections, which are imaged under a microscope. Fig. 1.2(a) shows a diagram of a cotton fiber cross section, while Fig. 1.2(b) shows a microscopic image of several cross sections. Then, using image analysis techniques [6], two measurements are taken from each cross section: the outer perimeter, $\mathrm{P}$, and the area of the secondary cell wall, A. Maturity, $\theta$, is then calculated according to

$$
\theta=\frac{4 \pi A}{P^{2}}
$$

which is the ratio of the area A to the area of a circle having perimeter $\mathrm{P}$.

Since sample preparation for the direct method is tedious and time consuming, other instruments have been developed that measure maturity indirectly. The Uster Advanced Fiber Information System (AFIS) uses an aeromechanical separator to individualize fibers and present them to an electro-optical sensor, where maturity is estimated based on the amount of light that is able to penetrate the fiber [7]. Also, the Cottonscope, measures maturity by analyzing the birefringence properties of $700 \mu \mathrm{m}$ fiber snippets under polarized light [8], [9].

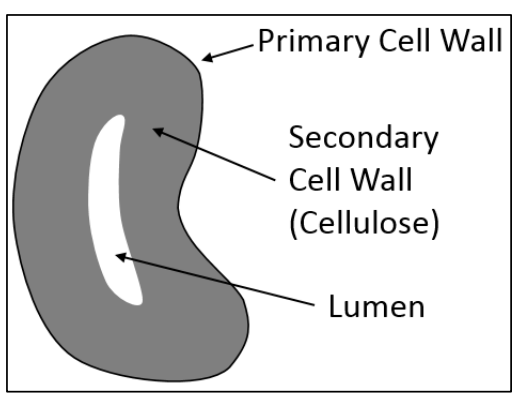

(a)

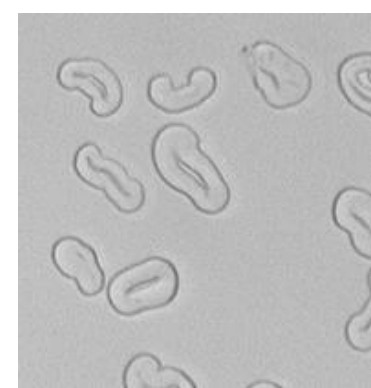

(b)

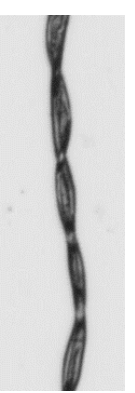

(c)

Fig. 1.2. (a) Diagram of the cross section of a cotton fiber. (b) Microscopic image of cotton fiber cross sections. (c) Image segment of a cotton fiber from the system described by [8]. 
In a precursor to our work, Shahriar et al. [10] established a proof-of-concept prototype designed to increase efficiency by measuring both length and maturity simultaneously from longitudinal images of individual fibers. Furthermore, as it has been shown that maturity can vary with a single fiber [11], the prototype system is uniquely capable of providing maturity measurements along the length of a fiber. Fig. 1.2(c) shows an image of a cotton fiber segment taken using this system. Actual images are much larger as they capture the entire length of the specimen.

While the goal is to generate a model that maps features, or measurements, extracted from the image to a maturity value, the difficulty lies in not knowing the maturities for the fibers being measured in the new instrument. For the prototype developed in [10], a single fiber is manually placed between two glass slides for imaging. Because the sample preparation for the direct method in [6] can only be applied to a bundle of fibers, it cannot be used to measure the maturity of a single fiber. However, the samples used in our study come from a set of reference cottons established by Hequet et al. [12]. Using these reference cottons, Shahriar et al. proposed a machine learning algorithm called G2DA for the purpose of transferring knowledge of maturity from the old instrument (cross section method) to the new instrument (image features). While the results show some success, the primary limitation of the G2DA algorithm is that it can only transfer knowledge when the input data of both measurement methods has the same dimensionality.

In this work we introduce a new transfer regression method that overcomes these limitations. By using a common link in the samples being measured by both instruments, we demonstrate that the probability distributions of labels from the older measurement method can be used to assist in the generation of a regression model to be used by the new instrument regardless of the dimensionality of the input data in either method. 


\section{Transfer Learning — Prior Work}

One problem faced by engineers and scientists in machine learning is that as data has become more abundant, labeling these data sets has become increasingly costly and time consuming. Transfer learning attempts to address this issue by presenting methods that leverage existing, often labeled, data sets (commonly called source domains) that relate to the primary learning task in some target domain. Relating this terminology to the task at hand, the older instrument would be considered the source domain and the new instrument would be the target domain, as indicated in Fig. 1.1.

Pan and Yang [13] divide transfer learning methods into three categories: inductive, transductive, and unsupervised. In this paper, we present methods that correspond to the transductive setting. This situation is primarily characterized by the fact that the source domain contains a lot of labeled data whereas the target domain has no labeled data. The transductive setting can be further divided into two categories: 1) the feature spaces between the source and target are different or 2) the feature spaces are the same but the probability distributions of the data are different. While many methods that have been developed address the latter (e.g. [14] and [15] as well as works cited by [13]), the former has received much less attention and is, perhaps, a more formidable problem. As previously mentioned, Shahriar et al. [10] proposed the G2DA algorithm, which provides a method of domain adaptation when the source and target domains are different. While G2DA is limited to both the source and target domains having the same dimensionality, some works have overcome this problem. Kulis et al. [16] describe a domain adaptation method extending prior work by Saenko et al. [17]. They demonstrate their method by classifying objects from different image domains (e.g. Speeded Up Robust Features (SURF) [18] in one domain and Scale Invariant Feature Transform (SIFT) [19] in the other) and report between $25-53 \%$ classification accuracy among various data sets - an improvement when compared to traditional unsupervised techniques. While that level of accuracy may sound relatively low, it illustrates how challenging this type of problem can be. 
Others include [20] and [21], both of which use co-occurrence data to establish a link between the source and target domains. While we use a similar concept to establish a relationship between the two instruments, their methods - along with [16] — do not apply to regression. Additionally, [20] and [21] assume there is some labeled data in the target domain, whereas ours does not.

One other aspect of transfer learning that has received little attention is regression. Yang et al. [22] propose a Bayesian multi-task learning model for nonlinear regression called HiRBF, where all learning tasks are combined in a single RBF network. Mao et al. [23] use the idea of principle curves to find a common lowdimensional representation across multiple tasks. Liu et al. [24] introduce Transfer Regression Model, which is based on manifold regularization, for the purpose of estimating indoor 3D location from Wi-Fi signals. Pardoe and Stone [25] employ the boosting technique and combine source and target data into one training set. More recently, Deng et al. [26] use generalized hidden-mapping ridge regression and apply it to both regression and classification in a transfer learning setting. Also, the aforementioned Shahriar et al. [10] use G2DA to transfer data from both source and target domains into a common latent space where least squares regression is applied. While these techniques present a wide variety of methods for transfer regression, none of these methods work when the dimensionality of the source and target domains are different. To our knowledge, no other transfer regression method can accomplish this - particularly when no labeled data exists in the target domain.

Also in this work, a novel feature selection algorithm is presented in the context of the transfer learning problem described above. Like regression, feature selection has not received as much attention in transfer learning research as classification. Methods proposed in [14] and [27] - [30] offer some examples; however, none of these approaches (nor any other to our knowledge) can be applied when the dimensionality of the source and target domains is different.

To summarize, the main contributions of this work are as follows: 
1. Introduce a novel transfer regression algorithm that can be used when the dimensionality of the source and target domains is different and when there is no labeled data in the target domain.

2. Introduce a novel feature selection algorithm that is applicable to the same scenario described above.

3. Using these new methods, demonstrate how they can be used to train a new instrument using information from an old instrument, particularly when both measurement methods, in essence, destroy the samples. 


\section{CHAPTER II METHODS}

As stated, the method presented here proposes to solve a transductive transfer learning regression problem. It is assumed there is one source domain representing to the old instrument in which all data is labeled (i.e. measurements are known for all samples) and one target domain representing the new instrument which contains data without labels (i.e. the samples analyzed by the new instrument do not have measurements). The learning task is to generate a regression model in the target domain of the new instrument that matches the way measurements are assessed in the source domain of the old instrument. To create a link between the two domains, we assume a single feature is common to both. Through this linked feature, this method utilizes distributions of measurements in both domains to perform the modeling.

\section{Formal Statement of the Problem}

What follows is an introduction of notation and definitions that frame the problem to be solved. Let $X^{S}=\left\{x_{1}^{S}, x_{2}^{S}, \ldots, x_{n_{S}}^{S}\right\}$ be a set of data points in the source domain, where $x_{i}^{S} \in \mathbb{R}^{d_{S}}, n_{S}$ is the number of data points, and $d_{S}$ is the number of dimensions. Let $Y^{S}=\left\{y_{1}^{S}, y_{2}^{S}, \ldots, y_{n_{S}}^{S}\right\}$ be a set of labels (i.e. measurements output from the old instrument) associated with each $x_{i}^{S}$ via some function $f^{S}: X^{S} \rightarrow Y^{S}$, where $y_{i}^{S} \in \mathbb{R}^{1}$. Furthermore, consider that $X^{S}$ is partitioned into $m_{S}$ classes or categories, $\mathcal{C}^{S}=\left\{c_{1}^{S}, c_{2}^{S}, \ldots, c_{m_{S}}^{S}\right\}$, such that $c_{j}^{S} \neq \varnothing$ for $j=1,2, \ldots, m_{S} ; \cup_{j=1}^{m_{S}} c_{j}^{S}=$ $X^{S}$; and $c_{j}^{S} \cap_{j \neq k} c_{k}^{S}=\emptyset$. With the possible exception of $f^{S}$, all source information is known and given.

Similarly, in the target domain, we are given a set of data, $X^{T}=$ $\left\{x_{1}^{T}, x_{2}^{T}, \ldots, x_{n_{T}}^{T}\right\}$, where $x_{i}^{T} \in \mathbb{R}^{d_{T}}, n_{T}$ is the number of data points in the target domain, and $d_{T}$ is the number of dimensions. These data are mapped to a set of unknown labels, $Y^{T}=\left\{y_{1}^{T}, y_{2}^{T}, \ldots, y_{n_{T}}^{T}\right\}$, where $y_{i}^{T} \in \mathbb{R}^{1}$, by some unknown function $f^{T}: X^{T} \rightarrow Y^{T}$. Also, just as in the source domain, $X^{T}$ is partitioned into $m_{T}$ classes, 
$\mathcal{C}^{T}=\left\{c_{1}^{T}, c_{2}^{T}, \ldots, c_{m_{T}}^{T}\right\}$, such that $c_{j}^{T} \neq \emptyset$ for $j=1,2, \ldots, m_{T} ; \cup_{j=1}^{m_{T}} c_{j}^{T}=X^{T}$; and $c_{j}^{T} \cap_{j \neq k} c_{k}^{T}=\varnothing$. The learning objective is to determine $f^{T}$. Note that both the dimensionality of the data as well as the number of data points in each domain can be different, i.e. $d_{S}$ is not necessarily equal to $d_{T}$, and $n_{S}$ is not necessarily equal to $n_{T}$.

The partitioning of the data in both the source and target domains is the key to relating the two domains. It is assumed that a relationship exists between $c_{j}^{S}$ and $c_{j}^{T}$ such that each category contains a set of features in its respective domain for the same group of related objects noted as $B_{j}$, i.e. $c_{j}^{S} \subset B_{j}$ and $c_{j}^{T} \subset B_{j}$. Intuitively, consider that the two domains share a categorical variable which can be used to group the data similarly in each domain. For the learning task, the number of categories is assumed to be the same in each domain, i.e. $m_{T}=m_{S}=m$. Additional categories may exist in one domain without a counterpart in the other. However, this data would not be useful for the learning task and is not considered.

\section{Feature Selection}

As with any machine learning problem, some features to be used in the learning task may be redundant or irrelevant. Before immediately trying to find $f^{T}$, it is typically desirable to determine which features in the target domain might be most useful and which ones offer no discriminating power. Since the target domain contains no labeled data, the proposed approach relies on relationships present among the various categories in the source domain and assumes similar relationships among the categories in the target domain. Maximum mean discrepancy (MMD) [31], [32] is used to quantify these relationships.

MMD is a metric in the space of probability distributions that estimates the difference in the means of two distributions. The main idea is that the distributions are embedded in a reproducing kernel Hilbert space (RKHS). There, the distance between distributions can be measured by the difference of their empirical means. The empirical estimate of the MMD between samples $\left\{x_{1}, \ldots, x_{m}\right\}$ and $\left\{z_{1}, \ldots, z_{n}\right\}$ is 


$$
M M D[X, Y]=\left\|\frac{1}{m} \sum_{i=1}^{m} \phi\left(x_{i}\right)-\frac{1}{n} \sum_{i=1}^{n} \phi\left(z_{i}\right)\right\|_{\mathcal{H}},
$$

where $\|\cdot\|_{\mathcal{H}}$ is the RKHS norm and $\phi$ is a kernel-induced feature map. It can be shown that, when $\mathcal{H}$ is universal, the MMD is zero if and only if the two distributions are the same [31]. One of the benefits of the MMD is that unlike other distance measures between distributions such as the Kullback-Leibler divergence, MMD does not require a density estimate. Although the cost of computation is $O\left((m+n)^{2}\right)$, randomization techniques can be employed to reduce this cost (e.g. bootstrapping).

Let $d$ be a measure that describes the distance, or separability, between categories in a given domain. In other words, $d\left(c_{j}^{Z}, c_{k}^{Z}\right)$ measures the distance between categories $c_{j}^{Z}$ and $c_{k}^{Z}$ for domain $Z$, where $Z=\{S, T\}$. This measure is defined as

$$
d\left(c_{j}^{Z}, c_{k}^{Z}\right)=M M D\left[P\left(x \mid c_{j}^{Z}\right), P\left(x \mid c_{k}^{Z}\right)\right]
$$

where $P\left(x \mid c_{k}^{Z}\right)$ is the probability distribution of the samples, $x$, for category $c_{k}^{Z}$. For notational convenience, the result of (2.1) is simply denoted as $d_{j k}^{Z}$.

Assuming the features in the target domain capture the same information as those in the source domain, then we also assume that the relative distances among the categories for each domain would be identical, which would happen in the extreme case where $X^{T}=X^{S}$. However, in practice this is not likely. In fact, in a transfer learning setting, it is reasonable to assume that little is known regarding the applicability of individual target domain features for predicting the desired dependent variable. This is especially true if the target domain differs significantly from the source domain, which will be a case presented later. Therefore, the objective is to choose a set of target domain features in order to minimize the difference between domains in the relative distances among the categories. In the remaining discussion, it is assumed that the source domain is well studied and that the associated features can 
be considered an optimal set for predicting labels $Y^{S}$. That is to say, the focus is solely on selecting features in the target domain.

For a chosen set of features $\Omega$ in the target domain, let a criterion function be defined as

$$
J_{\Omega}(r)=\sum_{k=1, k \neq r}^{m}\left(\frac{d_{r k}^{S}}{d_{r, \max }^{S}}-\frac{d_{r k}^{T}}{d_{r, \max }^{T}}\right)^{2},
$$

where $r$ is a chosen reference category and

$$
d_{r, \max }^{Z}=\max _{k=1 . . m}\left(d_{r k}^{Z}\right)
$$

is a normalization factor such that the distances in each domain are linearly scaled between 0 and 1 . Using the source domain as a guide, the reference category $r$ should be chosen as one of the two categories that are separated by maximum distance. In other words, let

$$
\left(r_{j}, r_{k}\right)=\underset{j, k=1 . . m}{\arg \max } d_{j k}^{S}
$$

then $r$ can be either $r_{j}$ or $r_{k}$.

The goal of this feature selection process is to choose a subset, $\Omega^{*} \subseteq \Omega^{T}$, that provides a balance between minimizing $J_{\Omega}$ and minimizing the total number of features in $\Omega^{*}$. One way to do this is to use Sequential Backward Selection (SBS) [33]. The steps for this algorithm are provided in Algorithm 2.1. Each time through the while loop, the algorithm removes one feature from $\widehat{\Omega}$ that minimizes $J_{\Omega}$ and adds it to the ordered list of features that have been discarded $\Omega^{-}$until only one feature remains. The order in which features are removed coupled with the value of $J_{\Omega}$ at each iteration provides information that can later be used to select $\Omega^{*}$. This idea is very similar to feature reduction using principal component analysis. There, eigenvectors are selected to strike a balance between minimizing the number of features while keeping a large amount of variance in the data. From the algorithm listed, $J^{d_{T}}$ can be 
analyzed along with $\Omega^{-}$to decide which feature subsets either minimize $J_{\Omega}$ or strike a balance between minimizing $J_{\Omega}$ and minimizing the total number of features. Note that $\Omega^{-}$will contain an ordered list of features from worst to best.

Algorithm 2.1. Feature Selection

Input: (1) List of target features $\Omega^{T}$

(2) Source and target data

Output: (1) Ordered list of features removed, $\Omega^{-}$

(2) $J^{d_{T}}$, a $d_{T}$-element array where $d_{T}=\left|\Omega^{T}\right|$

$1.1 \hat{\Omega} \leftarrow \Omega^{T}$

$1.2 \Omega^{-} \leftarrow \varnothing \quad$ //an ordered list of discarded features

$1.3 J^{d_{T}} \quad$ //initialize to zeros

$1.4 J^{0} \leftarrow$ eval $J_{\widehat{\Omega}}$

$1.5 \quad i \leftarrow 1 \quad$ //loop counter

$1.6 \quad$ while $|\hat{\Omega}|>1$

1.7 for each $\omega \in \hat{\Omega}$

$1.8 \quad \Omega^{\prime} \leftarrow \hat{\Omega} \backslash\{\omega\}$

$1.9 \quad$ eval $J_{\Omega^{\prime}}$

//keep track of lowest $J_{\Omega^{\prime}}$ and the

$/ /$ associated $\omega$

1.10 end

$1.11 \Omega^{-} \leftarrow \Omega^{-} \cup\left\{\omega_{\min }\right\} / /$ where $\omega_{\text {min }}$ minimized $J_{\Omega^{\prime}}$ above

$1.12 \hat{\Omega} \leftarrow \hat{\Omega} \backslash\left\{\omega_{\min }\right\} \quad / /$ remove $\omega_{\text {min }}$

$1.13 \quad J^{i} \leftarrow J_{\Omega^{\prime}, \min } \quad / /$ where $J_{\Omega^{\prime}, \min }$ is $J_{\Omega^{\prime}}$ associated with $\omega_{\text {min }}$

$1.14 \quad i \leftarrow i+1$

1.15 end

$1.16 \Omega^{-} \leftarrow \Omega^{-} \cup \widehat{\Omega} \quad$ //add the last feature

\section{Modeling the Target Domain}

The next task is to find a model that maps the target domain features to a label, which is the ultimate goal. Given a sample from the target domain, $x^{T}$, which has been analyzed by the new instrument, we want to find a function, $f^{T}$, that produces a label, $y^{T}$ : 


$$
y^{T}=f^{T}\left(x^{T}\right) .
$$

(For notational convenience, henceforth, $f^{T}$ will simply be denoted as $f$.) As stated above, for the source domain, the set of data points, $X^{S}$, and their associated labels, $Y^{S}$, are given. Furthermore, we also know the class or category to which each sample belongs. With this information we can estimate the probability distribution of the labels for a given category in the source domain, i.e. $P\left(y^{S} \mid c_{k}^{S}\right)$ for $k=1 . . m$. If $f$ is a good model for the labels in the target domain, then it stands to reason that the class conditional distribution of the labels output by one instrument should be (nearly) the same as the class conditional distribution of the labels from the other instrument. In other words, the following relationship is assumed

$$
P\left(y^{S} \mid c_{k}^{S}\right) \approx P\left(f\left(x^{T}\right) \mid c_{k}^{T}\right) \forall k=1 . . M .
$$

This is a reasonable assumption, since samples from $c_{k}^{S}$ and $c_{k}^{T}$ have both been drawn from the same group of related objects $B_{k}$. Furthermore, we assume those samples have been drawn in such a way that the sampling method for each domain produces a distribution that represents the population label distribution of $B_{k}$.

Initially, the difficulty in learning a model for the target space is that the available training data is unlabeled. In other words, samples analyzed by the new instrument do not yet have known output measurements. All that is known relating to labels in the target space are the class conditional distributions of the source data and the assumed relationship described by (2.3). While knowing only the distribution of labels for a given class is not immediately useful for the learning task, we can use the mean label of each class to seed the target space with an initial set of labels. Therefore, let $\dot{y}_{i}^{T}$ denote the initial label associated with $x_{i}^{T}$-the $i$ th sample in the target feature space. This initial label is assigned according to the following equation

$$
\dot{y}_{i}^{T}=E\left[y^{S} \mid c_{k}^{S}\right],
$$


given that the corresponding sample is an element of the $k$ th category, i.e. $x_{i}^{T} \in c_{k}^{T}$. In other words, the target label is initialized with the mean label value for category $c_{k}^{S}$. Assuming the distribution of data for each class is different $\left(P\left(x^{T} \mid c_{i}^{T}\right) \neq P\left(x^{T} \mid c_{j}^{T}\right)\right.$ for $i \neq j)$ and the mean label for each class is different $\left(E\left[y^{S} \mid c_{i}^{S}\right] \neq E\left[y^{S} \mid c_{j}^{S}\right]\right.$ for $i \neq$ $j$ ), the idea is that there will be enough label information available in the target space for a learning algorithm to get a general sense of how labels are distributed in the feature space and begin learning a model.

Because of this initialization step, the number of classes is important. If there are too few, the underlying function may not be recoverable. For example, just as in a simple regression case, one cannot expect to recover a quadratic function with only two data points. Similarly, in this method, one would not expect to uncover a quadratic function with only two classes as this would results in only two initial label values applied to all available target data.

Algorithm 2.2 shows the steps of the modeling process. Starting with the seeded target labels (Lines 2.2-2.4), the algorithm iteratively alternates between adjusting the target labels by applying a nonlinear map to the label values (Line 2.7) and learning a regression model using any appropriate method for the task (Line 2.8). At each iteration, label values for each data point in the target space are re-assigned (Line 2.9) in order to improve the next regression model until no further change is detected. The next section provides additional details and reasoning for this process. 
Algorithm 2.2 Modeling the Target Space

Input: (1) Source label distributions $P\left(y^{S} \mid c_{j}^{S}\right)$

(2) Target data $X^{T}$

(3) Categories $\mathcal{C}^{T}, \mathcal{C}^{S}$

Output: Model $g$

$2.1 \quad M_{\text {old }} \leftarrow \operatorname{Inf}$

//label initialization:

$2.2 \dot{y}_{i}^{T} \leftarrow E\left[y^{S} \mid c_{k}^{S}\right] \quad \forall i=1 \ldots n_{T}$

$2.3 g \leftarrow$ Regression $\left(X^{T}, \hat{Y}^{T}\right)$ //initial model

$2.4 Y^{T} \leftarrow g\left(X^{T}\right) / /$ reassign target labels based on $g$

$2.5 M_{\text {new }} \leftarrow \operatorname{eval} M$

2.6 while $\left|M_{\text {old }}-M_{\text {new }}\right|>\epsilon$

$2.7 \quad Y^{T} \leftarrow$ HistogramMatching $\left(Y^{T}, \mathcal{C}^{T}, Y^{S}, \mathcal{C}^{S}\right)$

$2.8 \quad g \leftarrow$ Regression $\left(X^{T}, Y^{T}\right) / /$ model

$2.9 \quad Y^{T} \leftarrow g\left(X^{T}\right) / /$ reassign labels

$2.10 \quad M_{\text {old }} \leftarrow M_{\text {new }}$

$2.11 \quad M_{\text {new }} \leftarrow \operatorname{eval} M$

2.12 end

\section{Modeling Algorithm Details}

Let $f(x)$ be the true, unknown model and $g(x)$ be the result of a regression model using the initialized labels, i.e. Lines 2.2-2.4 of Algorithm 2.2. Initially, the error between $g$ and $f$ is likely to be large. However, suppose a transformation, $H$, exists that maps the output of $g(x)$ directly to $f(x)$, e.g.

$$
f(x)=H(g(x)) .
$$

In a practical setting, finding an exact mapping is unlikely, however the idea is to condition the data such that the error in the estimate provided by $g$ is removed in order to improve the model in subsequent iterations. For this purpose, we have chosen to use histogram matching [34] as the transformation, $H$. Histogram matching is a process where values are reassigned via a nonlinear, monotonic process in order to match a 
specified histogram. Its purpose in the proposed algorithm is to force the distribution of labels for a given category in the target domain to match the distribution of labels for the same category in the source domain thereby reinforcing the knowledge transfer at each iteration. Histogram matching is a logical choice as it directly imposes the relationship in (2.3).

Prior to applying histogram matching, the label distributions in both domains must first be estimated. Histograms, like other nonparametric techniques, can estimate the underlying probability density function provided that the number of data points and the bin size are selected judiciously. As described by Theodoridis and Koutroumbas [35], for a fixed number of data points, the smaller the bin size, the higher the variance in the estimate. Larger bin sizes smooth out local variations in density. For a fixed bin size, the variance decreases as the number of sample points increases. For large enough number of samples, the smaller the bin size the better the accuracy of the resulting estimate.

With that in mind, the histogram can be fine-tuned prior to running the algorithm by selecting these two parameters for the source label distributions as these distributions are known and remain constant throughout the process. Assuming that the number of sample points is large enough, the choice of bin size for the source histograms is then assumed to be the same for the target label distributions in order to perform histogram matching.

Given that histogram matching is monotonic, we are able to examine conditions under which the algorithm can successfully recover $f$. Beginning with the ideal case in (2.5), taking the gradient of both sides results in

$$
\nabla f(x)=\nabla H(g(x))
$$

Since the gradients are equal, the signs of the gradients are also equal, i.e.

$$
\operatorname{sgn}(\nabla f(x))=\operatorname{sgn}(\nabla H(g(x)))
$$

where $\operatorname{sgn}()$ is the signum function, 


$$
\operatorname{sgn}(x)=\left\{\begin{array}{cc}
-1 & \text { if } x<0 \\
0 & \text { if } x=0 \\
1 & \text { if } x>0
\end{array}\right.
$$

Furthermore, since $H$ is monotonic,

$$
\operatorname{sgn}(\nabla H(g(x)))=\operatorname{sgn}(\nabla g(x))
$$

and, therefore,

$$
\operatorname{sgn}(\nabla f(x))=\operatorname{sgn}(\nabla g(x))
$$

This result implies that in the ideal case for $H$ to map directly from $g$ to $f$, the signs of the gradients of $g$ and $f$ must match. While this is a necessary condition for the ideal case, this is not realistic in practice.

Consider a neighborhood bounded by two points, $x_{1}$ and $x_{2}$, within which $f$ and $g$ can be approximated by a straight line. Furthermore, consider that within this neighborhood (2.6) does not hold. Fig. 2.1 shows a simple 1D case of this scenario, where $f\left(x_{1}\right)>f\left(x_{2}\right)$ while $g\left(x_{1}\right)<g\left(x_{2}\right)$. Let $\varepsilon$ be the total error at these two points between the true model and the training labels adjusted by histogram matching, i.e.

$$
\varepsilon=\left|H\left(g\left(x_{1}\right)\right)-f\left(x_{1}\right)\right|+\left|H\left(g\left(x_{2}\right)\right)-f\left(x_{2}\right)\right| .
$$

Since $H$ is monotonic, then $H\left(g\left(x_{1}\right)\right) \leq H\left(g\left(x_{2}\right)\right)$, and (2.6) is minimized when $H\left(g\left(x_{1}\right)\right)=H\left(g\left(x_{2}\right)\right)$. In the example in Fig. 2.1, this is equivalent to transforming $g$ into a horizontal line. In that case, let $y=H\left(g\left(x_{1}\right)\right)=H\left(g\left(x_{2}\right)\right)$, and the error becomes

$$
\varepsilon=\left|y-f\left(x_{1}\right)\right|+\left|y-f\left(x_{2}\right)\right| .
$$

By the triangle inequality, this implies that

$$
\varepsilon \geq\left|f\left(x_{2}\right)-f\left(x_{1}\right)\right| .
$$

In other words, the lower bound of the error is proportional to the slope of $f$. Note that we could arrive at this same result in the opposite case where $f\left(x_{1}\right)<f\left(x_{2}\right)$ and $g\left(x_{1}\right)>g\left(x_{2}\right)$. This means that for neighborhoods where (2.6) does not hold, the error is bounded by the expression in (2.8). Obviously, in the case where (2.6) is valid 
within the neighborhood, $f\left(x_{1}\right)>f\left(x_{2}\right)$, and $g\left(x_{1}\right)>g\left(x_{2}\right)$; then it is possible for both $H\left(g\left(x_{1}\right)\right)=f\left(x_{1}\right)$ and $H\left(g\left(x_{2}\right)\right)=f\left(x_{2}\right)$, which, from (2.7), results in $\varepsilon=0$.

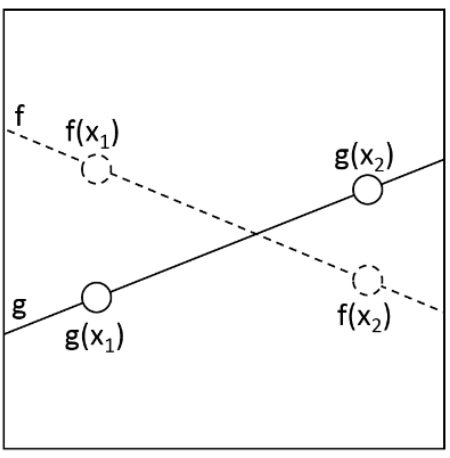

Fig. 2.1. Simple 1D example of a neighborhood where (2.6) does not hold.

As stated previously, the purpose of $H$ is to condition the data in order to improve the model at each iteration. If we consider $g\left(x_{i}\right)$ to be an estimate of $f\left(x_{i}\right)$, then the purpose of $H$ is to remove some of the error in the estimate before attempting to use that point to recover $f$. The analysis above indicates that $H$ can have better performance when the condition in (2.6) is met. In regions where (2.6) is not met, the modeling process is forced to use samples with potentially higher error. As with any regression problem, as noise increases over the space to be modeled, the resulting model can exhibit larger error. However, if the size or quantity of these regions is small, the errors are essentially smoothed out.

With this in mind, it becomes clear that the choice of model for $g$ is critical. While a particular model choice may not exactly match the true model $f$, as long as the condition in (2.6) is valid for a large portion of the relevant feature space, the final model can offer good approximation. In fact, a practical example of this scenario is provided in the results section.

While the analysis above provides some insight, (2.6) - (2.8) rely on $f$, which is unknown. However, we can derive some measures that indicate the performance of the modeling process using known information. Let $g_{k}(x)$ be the model generated by 
the algorithm in the kth iteration (Line 2.8), and let $H\left(g_{k-1}(x)\right)$ be the histogramadjusted labels of the model from the previous iteration (Line 2.7). We can calculate the root-mean-squared error of the modeling process at iteration $k$ by

$$
M=\left(\frac{1}{n_{T}} \sum_{i=1}^{n_{T}}\left(g_{k}\left(x_{i}\right)-H\left(g_{k-1}\left(x_{i}\right)\right)\right)^{2}\right)^{1 / 2} \text {. }
$$

This is the observable estimate of the true error-very similar to residuals in a typical regression sense. In practice, $M$ is a good indicator of convergence and is used as the stopping criteria, as indicated in Algorithm 2.2, Line 2.6. Upon termination, $M$ provides useful information in terms of the agreement between the final model $g_{k}$ and the labels suggested by the transfer of knowledge through $H$.

Note that $M$ is in the units of the desired output variable, which means acceptable values of the error indicated by $M$ are application dependent. However, we can use the coefficient of determination $R^{2}$ as a unitless measure of modeling performance. Ideally, both $g_{k}(x)$ and $H\left(g_{k-1}(x)\right)$ approach $f(x)$. In that ideal case, a scatter plot of $g_{k}(x)$ versus $H\left(g_{k-1}(x)\right)$ would reveal points along the diagonal $y=$ $x$. Assuming this model, we can then calculate $R^{2}$ by

$$
R^{2}=1-\frac{\sum_{i=1}^{n_{T}}\left(g_{k}\left(x_{i}\right)-H\left(g_{k-1}\left(x_{i}\right)\right)\right)^{2}}{\sum_{i=1}^{n_{T}}\left(H\left(g_{k}\left(x_{i}\right)\right)-E\left[H\left(g_{k-1}(x)\right)\right]\right)^{2}},
$$

where $E[\cdot]$ is the expectation operator. In the fraction above, the denominator remains relatively constant since $H$ enforces the relationship in (2.3). In other words, the variance in the data being modeled is generally the same at each iteration. As the model $g$ improves, the numerator decreases, causing an increase in $R^{2}$.

While either (2.9) or (2.10) could be used as stopping criteria, both provide a useful measure for model performance. As with a typical regression scenario, either could be used as criteria for model selection by repeating the algorithm for several different models and choosing the one that offers the best performance. 


\section{CHAPTER III RESULTS}

This section presents results and analyses for the feature selection and the modeling algorithms described above. First, a demonstration of the feature selection algorithm is presented on a set of synthetic data. Then, we present the modeling algorithm using two different examples based synthetic data and one example using real world data.

\section{Feature Selection}

For the first experiment, two sets of data are created, one each for the source and target domains. The source domain consists of six categories where each category contains 5000 data points randomly sampled from a bivariate normal distribution with different means but the same covariance, $\Sigma_{S}=\operatorname{diag}\left(\sigma_{S}^{2}, \sigma_{S}^{2}\right)$, where $\sigma_{S}$ is the chosen standard deviation. The target domain consists of the same six categories - each with 5000 points randomly sampled from a normal distribution in $\mathbb{R}^{3}$, also with different means but the same covariance, $\Sigma_{T}=\operatorname{diag}\left(\sigma_{T}^{2}, \sigma_{T}^{2}, \sigma_{T}^{2}\right)$, where $\sigma_{T}$ is the chosen standard deviation. Table 3.1 shows the means for each category in each domain. The means in each domain were chosen such that all features are required to separate each category in the respective feature space. Also, the target domain consists of three additional features that represent noise. Each of these noisy dimensions contains data randomly sampled from the unit normal, $\mathcal{N}(0,1)-5000$ points for each category. In total, the target domain consists of six features - three of which are "good" features and the other three are noise. Fig. 3.1 shows plots of the data in each domain. 
Table 3.1 Means of the Six Categories in the Source and Target Domains

\begin{tabular}{lcc}
\hline & Source Domain & Target Domain \\
\hline Category 1 & $(0,0)$ & $(0,0,0)$ \\
Category 2 & $(0,1)$ & $(0,0,1)$ \\
Category 3 & $(1,1)$ & $(0,1,1)$ \\
Category 4 & $(1,2)$ & $(1,1,1)$ \\
Category 5 & $(2,2)$ & $(1,2,1)$ \\
Category 6 & $(2,3)$ & $(1,2,2)$ \\
\hline
\end{tabular}

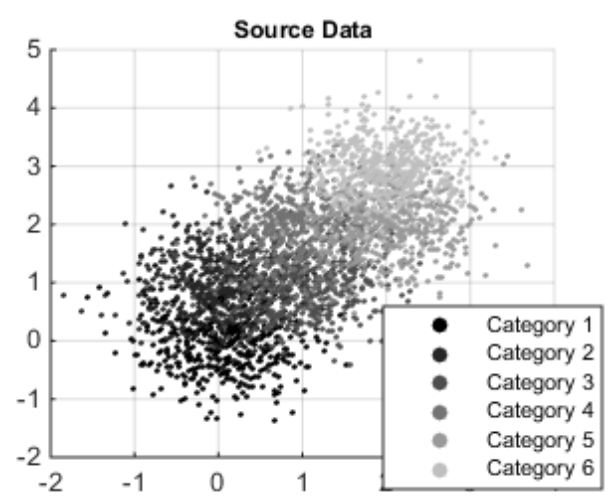

(a)

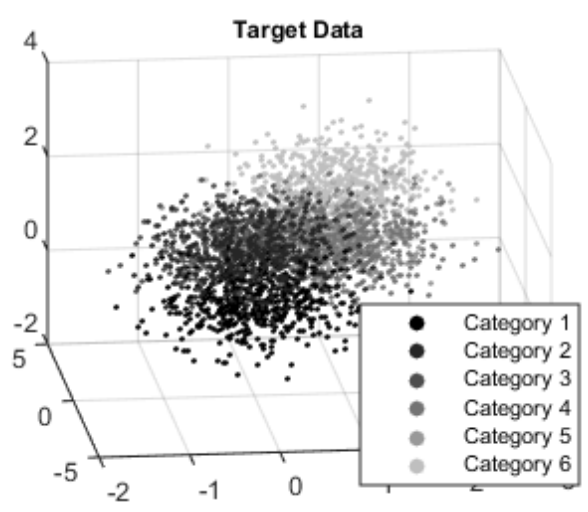

(b)

Fig. 3.1. Synthetic data used to test the feature selection algorithm. (a) Two source domain features. (b) Three good target domain features.

The objective of this experiment is twofold. First, we verify that the feature selection algorithm can identify the three good features in the target domain regardless of the order in which the features are presented. To accomplish this, the algorithm was run repeatedly on the synthetic data while randomly permuting the order of the target features each time. Since the algorithm produces an ordered list of features, $\Omega^{-}$, the good features are expected to appear as the last three in the list, i.e. the last three removed. Second, the effect of overlap among the categories in both the source and target domains is analyzed as well as the choice of bandwidth for the Gaussian kernel used in the MMD. (Bandwidth selection is made according to Gretton et al. [32], 
which suggests using the median distance between points in the aggregate sample of the two distributions being compared.) Results are presented showing how the standard deviations of the categories, $\sigma_{S}$ and $\sigma_{T}$, were varied in each domain separately. Finally, it should be noted that all data in both the source and target domains were normalized prior to each run such that each feature, independently, had a zero mean and unit variance.

Table 3.2 shows the results of the first experiment. For a given $\sigma_{S}$ and $\sigma_{T}$, the algorithm was run 20 times - each time with a new randomly generated set of data with the given parameters and with the target features randomly ordered. Inside each table cell, the three numbers indicate how many of the last three features output by the algorithm were the known good features: "exactly three / exactly two / exactly one." For example, the result 18/1/1, means 18 of the runs were an ideal result (the three good features were the last three removed), one run found two of the good features in the last three listed, and one run only found one good feature. A perfect result is 20/0/0.

Fig. 3.2 shows some examples of the behavior of the criterion function (2.2) during the feature selection process. Each line is an example of one run of the algorithm taken from each batch in the second row of Table 3.2 where $\sigma_{S}=1$. Each of these produced an ideal result, meaning the good features were the last to be removed. While the minimum does not necessarily occur when the third feature is removed in each case, in most cases it is clear that when the fourth feature is removed, the value of the criterion function begins to sharply rise. The obvious exception to this is when $\sigma_{T}=0.5$, which is a case discussed above.

While the feature selection method operates independently of the modeling algorithm, they are both predicated on the same assumptions regarding the problem setting - i.e. the dimensionality of the source and target are different and there are no labels in the target domain. As feature selection is often an important part of a machine learning problem, the method presented here provides information that can be 
used in the modeling step. In the case study presented later, we show how these two methods can work together.

Table 3.2 Feature Selection - Synthetic Data Results

\begin{tabular}{|c|c|c|c|c|c|}
\hline \multicolumn{3}{|c|}{ Target, $\boldsymbol{\sigma}_{\boldsymbol{T}}$} & \multirow[b]{2}{*}{2} & \multirow[b]{2}{*}{4} & \multirow[b]{2}{*}{8} \\
\hline Source, $\sigma_{S}$ & 0.5 & 1 & & & \\
\hline 0.5 & $20 / 0 / 0$ & $20 / 0 / 0$ & $20 / 0 / 0$ & $20 / 0 / 0$ & $4 / 5 / 11$ \\
\hline 1 & $11 / 9 / 1$ & $20 / 0 / 0$ & $20 / 0 / 0$ & $20 / 0 / 0$ & $11 / 9 / 0$ \\
\hline 2 & $0 / 20 / 0$ & $20 / 0 / 0$ & $20 / 0 / 0$ & $20 / 0 / 0$ & $14 / 6 / 0$ \\
\hline 4 & $0 / 18 / 2$ & $20 / 0 / 0$ & $20 / 0 / 0$ & $20 / 0 / 0$ & $13 / 7 / 0$ \\
\hline 8 & $0 / 19 / 1$ & $17 / 3 / 0$ & $18 / 2 / 0$ & $17 / 3 / 0$ & $12 / 7 / 1$ \\
\hline
\end{tabular}

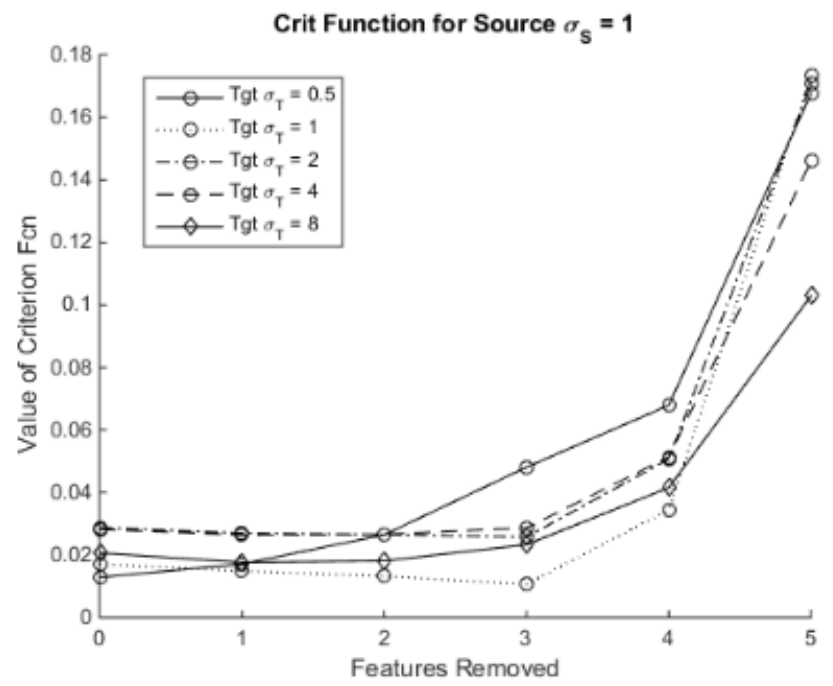

Fig. 3.2. An example of the behavior of the criterion function (2.2). Each line is an example of one run of the algorithm taken from each batch in the second row of Table 2 where $\sigma_{S}=1$.

\section{Modeling the Target Domain}

In this section we present three examples that demonstrate the effectiveness of the proposed modeling algorithm.

\section{D Synthetic Data}

For illustrative purposes, let us begin with a simple 1D example. Consider that a domain in $\mathbb{R}^{1}$ consists of three categories of 100 samples each, and each category is 
normally distributed as $\mathcal{N}(\mu=-1, \sigma=1), \mathcal{N}(0,1)$, and $\mathcal{N}(1,1)$. Also, let the true model in this domain be quadratic, $y=3 x^{2}$. In a typical problem setting, this model is unknown and the goal is to recover it from the given information. First, one set of data is randomly generated according to the above parameters. Using this data, the class conditional distributions of the labels are computed and treated as if they are obtained from a source domain, i.e. $P\left(y^{S} \mid c_{k}^{S}\right)$. Then a new set of data is randomly generated using the same parameters. This data set is treated as the target domain data and is shown in Fig. 3.3(a) with the true labels. The first step of the algorithm is to initialize the labels according to (2.4), as shown in Fig. 3.3(b). Next, with a chosen model, a regression scheme is applied to the initialized data. For the purposes of a successful demonstration, we have chosen a quadratic model and are using a linear least squares regression method. Fig. 3.3(c) shows the result of the first modeling attempt. Now the iterative process begins with histogram matching where labels are reassigned to enforce the relationship in (2.3), as shown in Fig. 3.3(d). Because our initial model meets the condition described by (2.6) almost everywhere, immediately, the form of the true model can be seen after the transfer of knowledge through histogram matching. Next, using the histogram-adjusted labels, the same regression scheme is applied and labels are reassigned according to the new model (Fig. 3.3(e)). Figs. 3.3(f) and $(\mathrm{g})$ show the results of the second iteration. Finally, after five iterations, the algorithm terminates according to the stopping criteria $\epsilon=0.01$ (see Algorithm 2.2, Line 2.6). The final model is $y=3.29 x^{2}-0.44 x+0.03$. Figs. 3.3(h) and (i) show the values of $M$ and $R^{2}$ from equations (2.9) and (2.10), respectively, at each iteration. In these two graphs, Iteration 0 represents the values after initialization and the first model (Algorithm 2.2, Line 2.5), which is prior to the knowledge transfer by histogram matching. Given that we know the true labels in this example, the root mean squared error (RMSE) of the true labels versus the generated model is 0.89 , while the final value of $M$ is 1.51 . Also the final value of $R^{2}$ from (2.9) is 0.95 . 


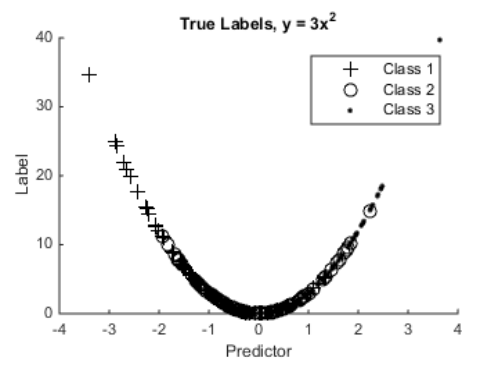

(a)

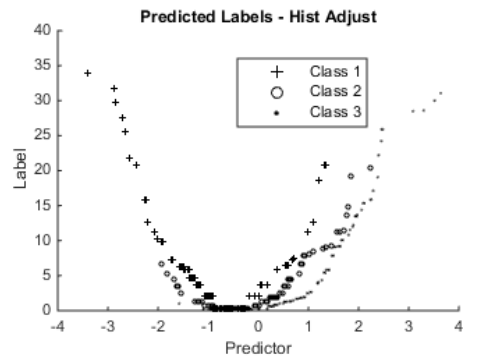

(d)

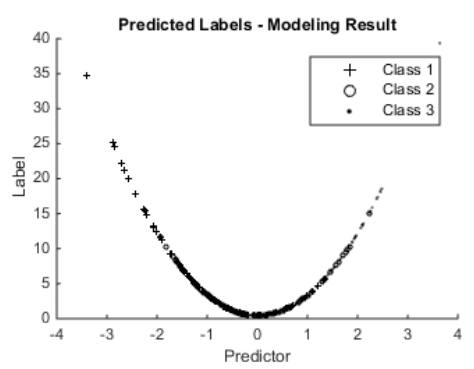

(g)

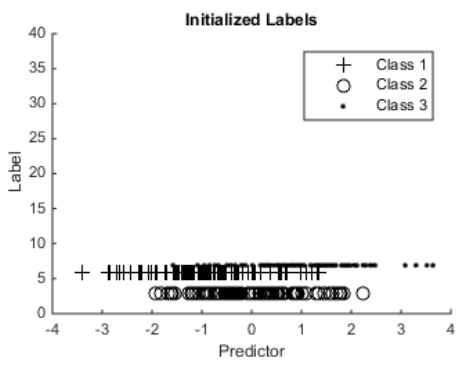

(b)

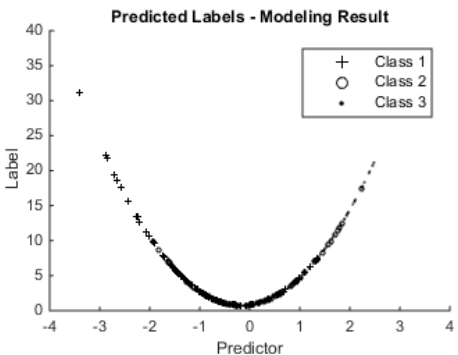

(e)

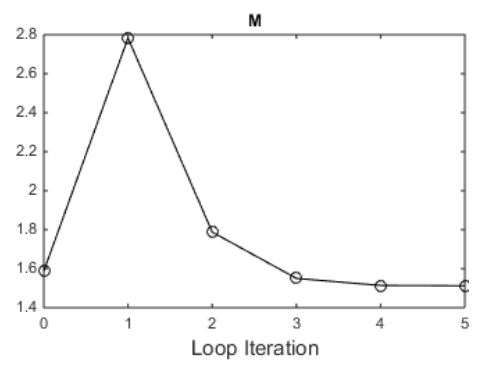

(h)

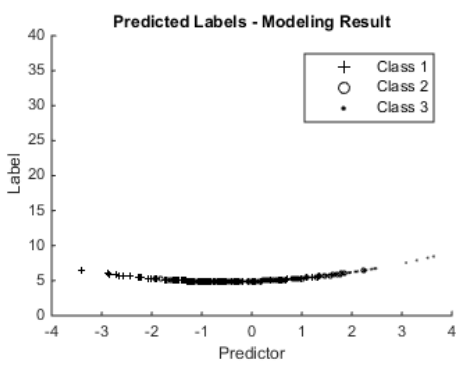

(c)

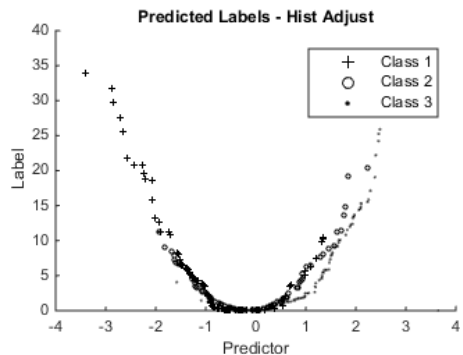

(f)

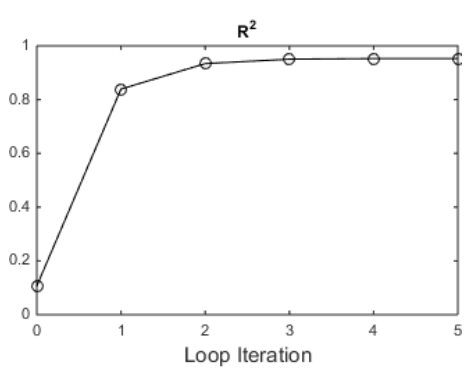

(i)

Fig. 3.3. Demonstration of modeling algorithm on 1D synthetic data with a quadratic model $y=3 x^{2}$. (a) Synthetic data of three categories with labels assigned by the true model. (b) Initialized labels according to (2.4). (c) Result of first modeling attempt. First iteration: (d) labels are reassigned via histogram matching and (e) modeling result after histogram matching. Second iteration: (f) histogram matching and (g) modeling. (h) Plot of $M$ (Eq. (2.9)) over the five iterations. (i) $R^{2}$ (Eq. (2.10)) over five iterations.

This simple example demonstrates that without any existing label information in the target space and, instead, given only a distribution of labels per category, the proposed algorithm can recover a model in the target space. In this case, the simulated 
source distributions were generated from distributions with a sample size of only 100 points each. By increasing those sample sizes to 1000 per category and rerunning the experiment, we get a final model $y=2.92 x^{2}+0.14 x+0.01$, true $\operatorname{RMSE}=0.27$, $M=0.59$, and $R^{2}=0.99$, which is a marked improvement.

Additionally, in order to analyze the bias of the procedure, we ran this experiment 50 times - each time sampling a new set of 1000 points per category with the above distribution parameters. Table 3.3 shows the results of the model parameters along with a $95 \%$ confidence interval (CI). While there exists some bias in the $x^{2}$ and constant coefficients, it results in a very small amount of error.

Table 3.3 1D Synthetic Example - Bias of the Estimator

\begin{tabular}{ccc}
\hline Coefficient & Mean & $95 \%$ CI \\
\hline$x^{2}$ & 2.9529 & {$[2.92482 .9928]$} \\
$x$ & 0.0073 & {$[-0.05150 .0661]$} \\
Constant & 0.0837 & {$[0.04440 .123]$} \\
\hline
\end{tabular}

Finally, using this synthetic example, we wish to illustrate the scenario when (2.6) does not hold. The procedure was run once more with the same parameters except this time the regression model was a simple linear model. Fig. 3.5 shows the results a few iterations into the procedure. In Fig. 3.4(a), the labels have been assigned values according to a new model at the end of one iteration (Line 2.9, Algorithm 2.2), and Fig. 3.4(b) shows the results of the histogram matching step at the beginning of the next iteration (Line 2.7, Algorithm 2.2). The dashed line represents the true model, $y=3 x^{2}$, while the dash-dot line is the simple linear model estimated thus far. The shaded region in each graph shows where the signs of the gradients between the two models do not match, i.e. (2.6) does not hold. It is clear from Fig. 3.4(b) that in the non-shaded region, the histogram matching step results in labels that bear some resemblance to the true model. However, in the shaded region, histogram matching tends to transform the data into a horizontal line; which, as stated in the 
discussion between (2.6) and (2.8), is the best histogram matching can do when (2.6) does not hold.

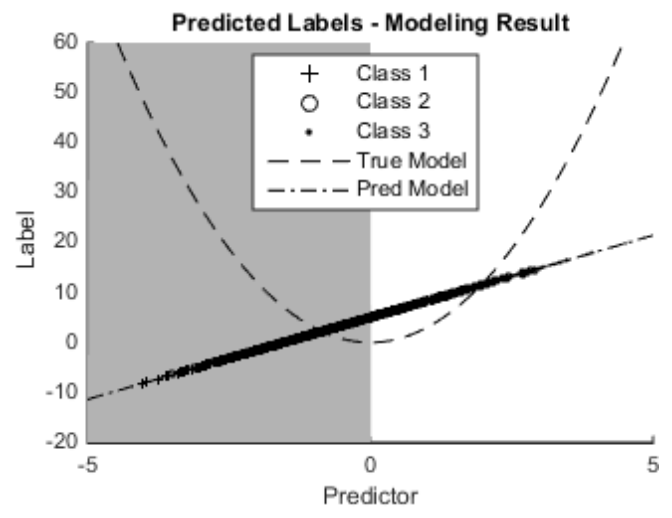

(a)

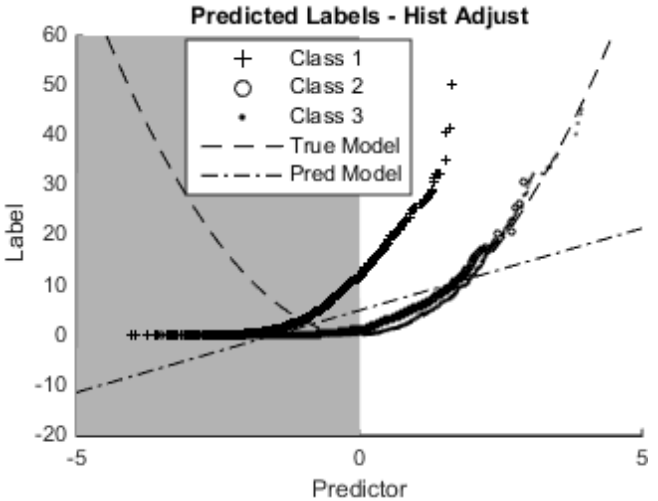

(b)

Fig. 3.4. An illustration of the scenario when (2.6) does not hold (shaded regions). (a) Labels are assigned according to a simple linear model. (b) Histogram matching is applied.

\section{D Synthetic Data}

As an additional demonstration of the algorithm on synthetic data, let us consider an example of 2D data with an affine transformation between the source and target domains. The source domain consists of five categories of 500 points each and normally distributed with means $(-2,2),(-1,1),(0,0),(1,-1),(2,-2)$ each and a standard deviation of 0.5 . Let the source domain model be a sigmoid function,

$$
y=\frac{1}{1+e^{-(z \prime x) / 2}},
$$

where $x \in \mathbb{R}^{2}$ and $z^{\prime}=\left[\begin{array}{ll}-1 & 1\end{array}\right]$. This source domain data is shown in Fig. 3.5(a) with the shade of each point indicating the label value. Additionally, a different set of samples is generated along with associated labels using the same parameters; however, an affine transformation is then applied to this second set of data according to

$$
x^{T}=\left[\begin{array}{cc}
2 & 1 \\
3 & -2
\end{array}\right] x+\left[\begin{array}{c}
2 \\
-1
\end{array}\right]
$$


where $x^{T}$ represents the location of sample in the transformed (target) space. The target data with the true labels is shown in Fig. 3.5(b). Following the initialization of labels in the target domain according to (2.4), Fig. 3.5(c) shows the data with five discrete label values. In this figure, the five categories are visually discernible. For the modeling algorithm, we choose logistic regression and apply the modeling procedure. After three iterations, the algorithm terminates with the following logistic function as the result

$$
y=\frac{1}{1+e^{-\left(0.0729 x_{1}-0.215 x_{2}-0.3487\right) / 2}}
$$

where $x_{1}$ and $x_{2}$ represent the first and second dimensions, respectively. Fig. 3.5(d) shows a plot of predicted labels versus true labels. The final results are true RMSE $=$ $0.002, M=0.006$, and $R^{2}=0.999$ indicating a very close match. Note that the true labels were generated from (3.1) prior to applying the affine transformation.

This second example illustrates two points. First, the algorithm is robust in that it can support different regression models and methods. No changes were required between the first and second examples other than to simply use a different type of regression model when specified in Algorithm 2.2, Line 2.8. Second, there are no assumptions nor requirements made on the distribution of samples in the source domain. In other words, there is no assumed relationship between $P\left(x^{S} \mid c_{j}^{S}\right)$ and $P\left(x^{T} \mid c_{j}^{T}\right)$. There is a significant amount of prior work that deals with domain adaptation (e.g. the aforementioned works [10], [13] - [17]), which often deals with finding a transformation that maps samples from the source to the target domain or from both domains to a latent space. Typically, this is done for the purpose of transferring labeled samples from the source to the target for the learning task. In the example presented here, the goal of domain adaptation could be attempting to recover the affine transformation in (3.2). However, as demonstrated, the proposed modeling algorithm does not require knowledge of any type of domain adaptation, and, instead, 
conducts the learning task directly in the target domain using only distributions of labels from the source, i.e. $P\left(y^{S} \mid c_{j}^{S}\right)$.

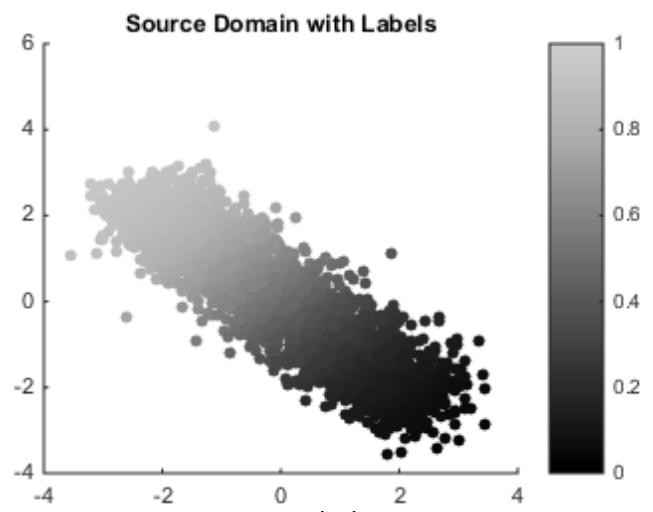

(a)

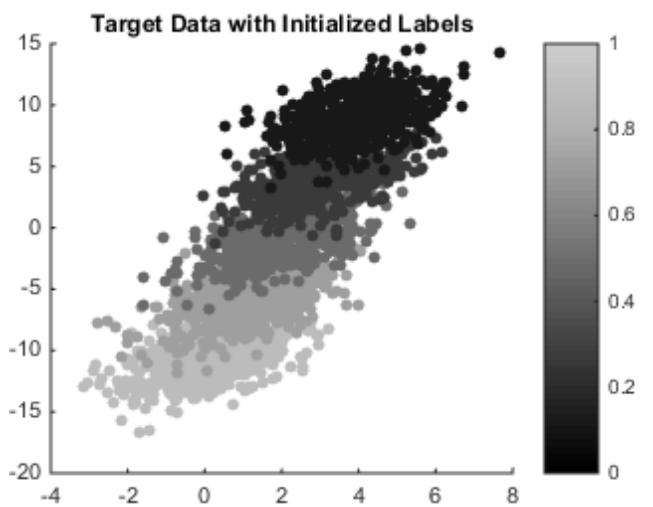

(c)

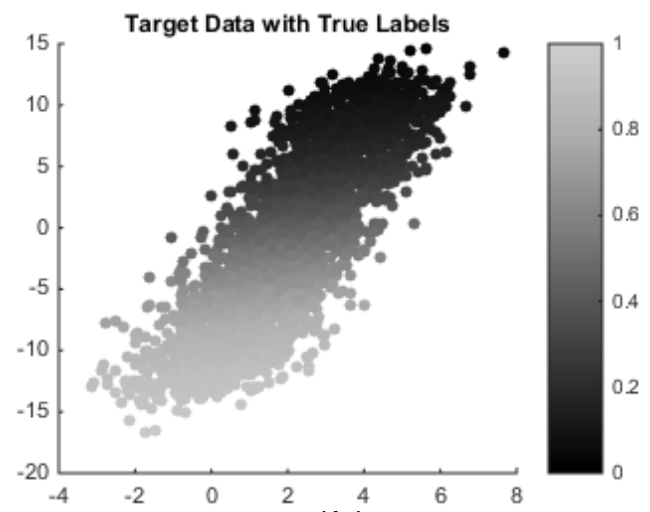

(b)

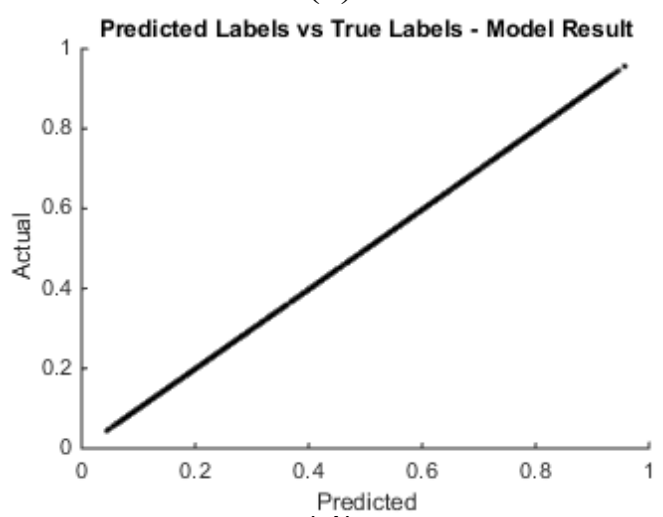

(d)

Fig. 3.5. A 2D example of synthetic data. (a) Source domain with the shade of the point relating to label value. (b) Target domain with true labels. (c) Target domain with initialized labels. (d) Plot of predicted versus true labels after completion of the algorithm.

Also, using this set of data, we analyzed the effects of divergence of the distributions between the source and true target labels. The modeling algorithm is based on a key relationship described by (2.4), which assumes that the data in the source and target domains were sampled in the same manner and, therefore, produce similar label distributions. In this particular variation of the example described above, the features are sampled from normal distributions with means at the same five locations mentioned previously and with the same variance. For the target domain 
data, a small amount of noise was added to each of these means with the noise being sampled from a uniform distribution over a specified interval for both dimensions (see Table 3.4 for a list of specific intervals). This has the effect of creating a set of target data with slightly different true label distributions than the source, i.e. it adds a bias to the target distributions. This experiment was run 20 times for each interval, and the final value of $M$ and $R^{2}$ was recorded after each. Table 3.4 shows the mean and standard deviation for $M$ and $R^{2}$ over these 20 runs for each interval. As the categories for the target domain are shifted by larger distances versus their source domain counterparts, clearly both the mean and standard deviation of the performance measures increase - meaning it becomes increasingly difficult for the modeling algorithm to match the source label distributions using noisy target data. However, considering that the output variable is on the range from 0 to 1 , for the largest mean shift interval, the mean value of $M$ is only 0.023 and $R^{2}$ is 0.99 indicating a very good match. Furthermore, it should be noted that the values of $M$ follow closely with the True RMSE indicating that $M$ is a good estimate of the true error.

Table 3.4 Adding Bias to the Target Distributions

\begin{tabular}{l|cl|cl|cc}
\multicolumn{2}{c|}{} & \multicolumn{2}{c|}{$R^{2}$} & \multicolumn{2}{c}{ True RMSE } \\
\hline \multicolumn{1}{c|}{ Interval } & Mean & Std. Dev. & Mean & Std. Dev. & Mean & Std. Dev. \\
\hline$[-0.01,0.01]$ & 0.006141 & 0.00075 & 0.999527 & 0.000116 & 0.002541 & 0.00129 \\
{$[-0.1,0.1]$} & 0.008343 & 0.001335 & 0.999117 & 0.000272 & 0.004549 & 0.002412 \\
{$[-0.25,0.25]$} & 0.014079 & 0.004891 & 0.997262 & 0.001837 & 0.016616 & 0.006832 \\
{$[-0.5,0.5]$} & 0.023489 & 0.008908 & 0.992232 & 0.005806 & 0.025004 & 0.010602
\end{tabular}

\section{Real World Data}

In the next experiment, we show how model selection can be performed and present a case where the model chosen for regression doesn't match the true model as it did in the previous example. Furthermore, this experiment utilizes cotton fiber maturity data made available by Hequet et al. in [12], which allows us to present an example based on a realistic scenario. 
The work in [12] produced maturity data for 104 cotton bales representing two principal cultivated species. For these bales, maturity was evaluated by the cross section method and the Cottonscope instrument. A sample was extracted from each bale according to an industry standard protocol described in [12] and measured by each method/instrument. The Cottonscope measures the average maturity of a fiber snippet $700 \mu \mathrm{m}$ in length and outputs a distribution of maturities for all snippets in a cotton sample. No other measurement information is available-just the output maturity distributions. However, for the cross section method, perimeter and area measurements are recorded for at least 4000 cross sections per bale. For this experiment, we are treating the cross section measurements as the target domain, while the Cottonscope measurement represents the source. In other words, suppose that the Cottonscope is the old instrument which we want to use to train a new instrument that uses the cross section method. The true model for the cross section method is given by (1.1), and the objective is to generate a model in the cross section domain that approximates the measurements of the Cottonscope instrumentassuming that in a more realistic scenario (1.1) would be unknown. From the data in [12], we have chosen to use five bales for training. These bales were selected to represent a broad range of maturity values. Fig. 3.6 shows the distribution for each bale. Also Fig. 8 shows a scatter plot of the data in the cross section domain, where the perimeter and area have been normalized. The shading in Fig. 3.7(a) shows the true maturity, while the shading in Fig. 3.7(b) shows maturity values after applying the target label initialization in (2.4). Note that in this second figure, it is difficult to visually differentiate the categories due to the high degree of overlap. 


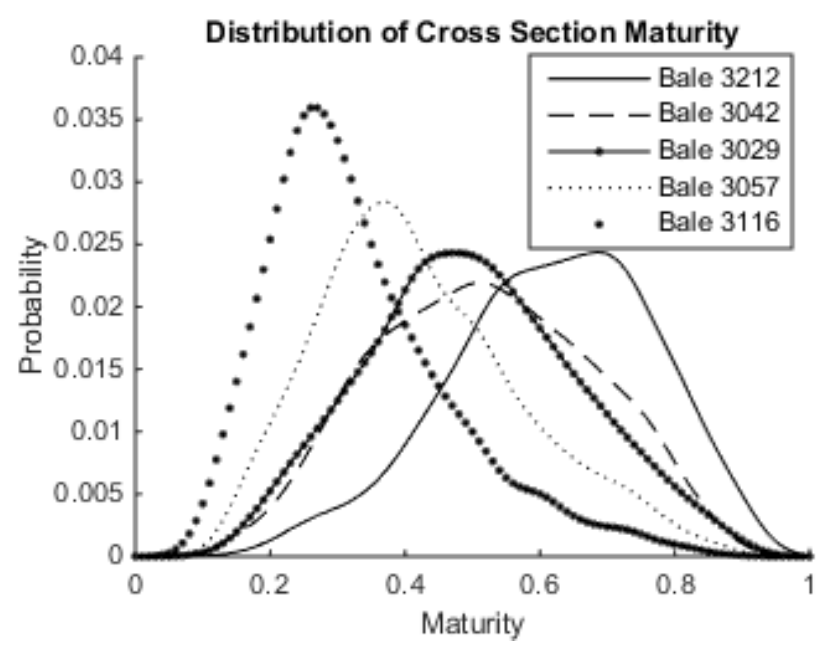

Fig. 3.6. Distribution of maturity for the five bales chosen as training data. The bale numbers in the legend $(3212,3042$, etc.) correspond to specific bales discussed by Hequet et al. [12].

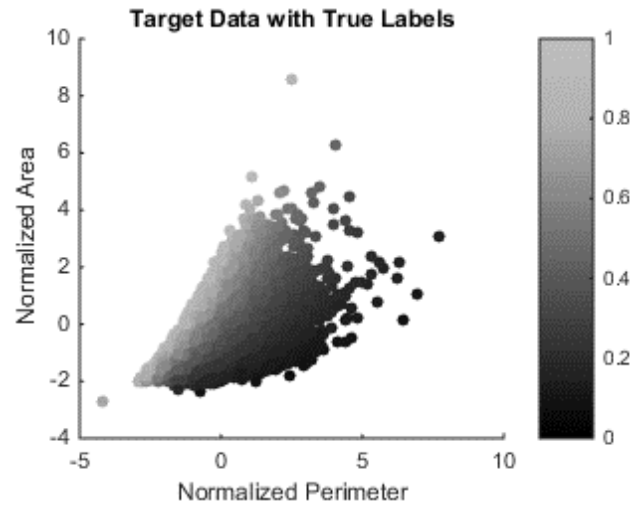

(a)

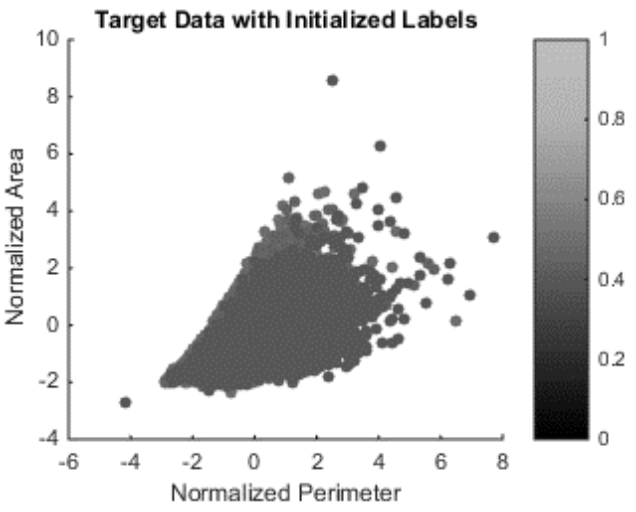

(b)

Fig. 3.7. Cotton fiber cross section data. (a) Target data with true labels. (b) Target data with initialized labels.

With this data, the algorithm was run for three different models: linear, quadratic, and logistic. The cross section data is split so that $80 \%$ is used for training and the remaining $20 \%$ is used for testing. Table 3.5 shows the results of each model. Out of these three, the quadratic model presents the lowest value of $M$ and the highest $R^{2}$, suggesting that this model would be the best out of the three. This is confirmed by the RMSE value in the right column, which is calculated from the true label values in 
the test data. Also, Fig. 3.8 shows a plot of predicted versus true labels for each model using the test set.

Table 3.5 Model Selection - Cross Section Data as Target Domain

\begin{tabular}{lccc}
\hline Model Type & $M($ Eq. (7)) & $R^{2}$ (Eq. (8)) & True RMSE \\
\hline Linear & 0.058 & 0.90 & 0.058 \\
Quadratic & $\mathbf{0 . 0 4 6}$ & $\mathbf{0 . 9 4}$ & $\mathbf{0 . 0 4 4}$ \\
Logistic & 0.052 & 0.92 & 0.048 \\
\hline
\end{tabular}

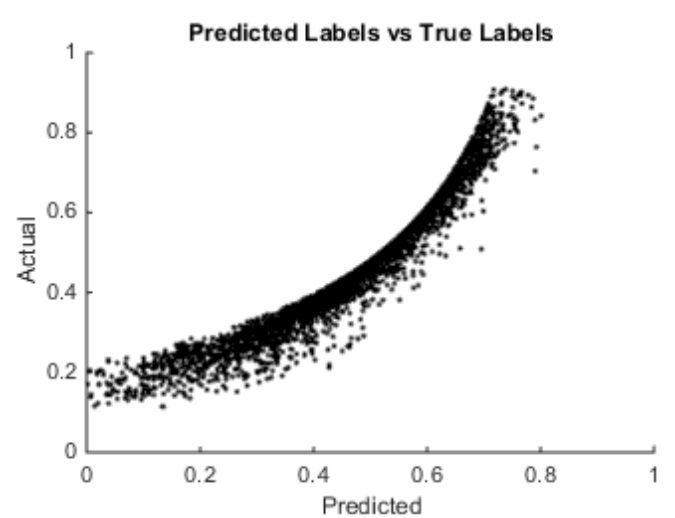

(a)

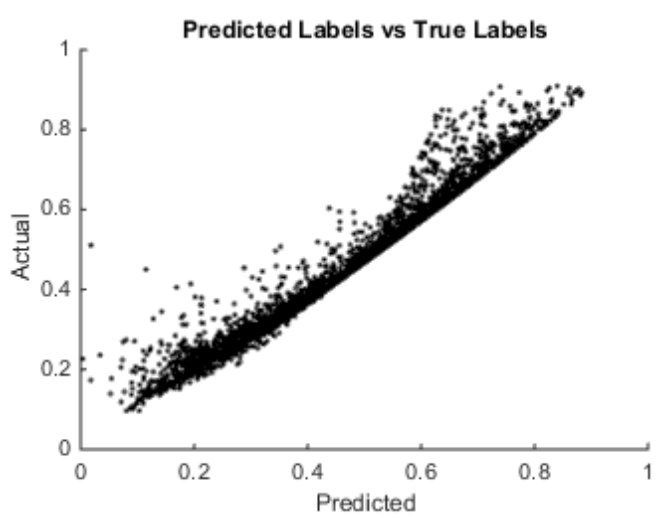

(b)

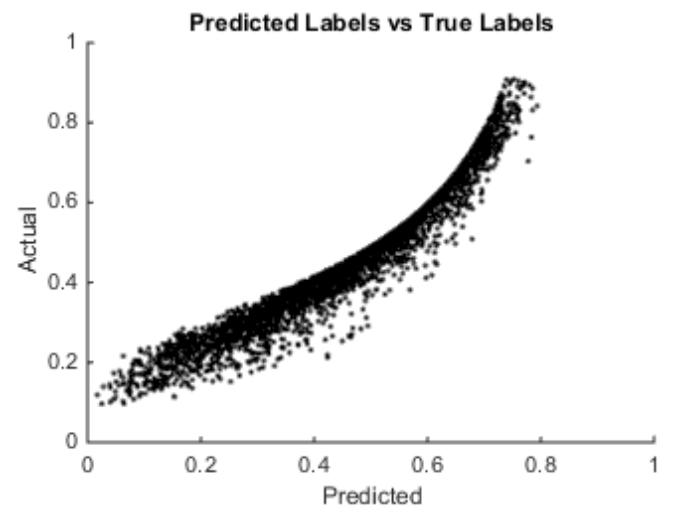

(c)

Fig. 3.8. Scatter plot of predicted labels versus true labels for each of the models attempted using the cotton fiber cross section data as the target. (a) Linear. (b) Quadratic. (c) Logistic. 
The results of this experiment demonstrate two important points. First, while the three models chosen do not exactly match the true model in (1.1), the quadratic model offers a close approximation exhibiting relatively low error. In many cases it may be impractical to determine the true underlying model; however, an approximation of that model can produce good results. Second, the measures proposed in (2.9) and (2.10) provide a good estimate of model performance when compared to known, true labels. In a more realistic scenario, no known labels would exist in the target domain making the reliability of these two measures necessary. 


\section{CHAPTER IV ESTIMATING COTTON FIBER MATURITY}

As stated in the introduction, the primary motivation for the methods described in this dissertation is to assess maturity using the line scan system proposed by Shahriar et al. [10]. Currently, with the prototype system, individual fibers are placed between two glass slides and then mounted onto a moving stage. As the stage is moved, a line scan camera captures the entire length of the specimen producing images that are approximately $8 \mathrm{k} \times 50 \mathrm{k}$ pixels with a $1 \mu \mathrm{m}^{2}$ per pixel spatial resolution. The images are processed to segment the fiber from the background and smaller images of fiber segments, called tiles, are extracted from one end of the fiber to the other. Although the size of each tile varies, they are generally on the order of 100x100 pixels and contain a fiber segment approximately $150 \mu \mathrm{m}$ in length. Furthermore, each tile overlaps the neighboring tiles by $50 \%$ - meaning a tile contains $50 \%$ of the segment belonging to the previous tile as well as $50 \%$ of the segment belonging to the next tile. Cotton fiber lengths vary from 2 to $4 \mathrm{~cm}$, so one image of a fiber can generate between 200 and 500 tiles. Fourteen measurements are extracted from each tile. Five features are based on the fiber width within the tile: fiber width minimum, maximum, average, standard deviation, and shape factor (the ratio of the maximum width to the minimum width). There are also four features based on the translucency (pixel intensity) of the fiber: intensity minimum, maximum, average, and standard deviation. Finally, there are four Haralick texture features [36]: contrast, correlation, energy, and homogeneity.

Given some cotton samples, the objective is to use maturity calculated from cross sections of cotton fibers to train this new system to estimate maturity using the fourteen features extracted from each tile. Relating this to a transfer learning setting, the cross sections represent the source domain and the longitudinal image features are the target domain. Samples for both domains are obtained from a set of cotton bales described by Hequet et al. [12], meaning the actual bales of cotton used there are also used in this study. While Hequet acquires cross section measurements from a total of 104 cotton bales, only 10 of those were scanned using the longitudinal line scan 
system. Of those 10, five bales were chosen as training data presented in this case study as they exhibited a broad range of maturity values. These are the same five bales used in the previous example indicated by Fig. 3.6. These density estimates are calculated based on approximately 4000 cross sections for each bale and represent the source domain, i.e. $P\left(y^{S} \mid c_{j}^{S}\right)$. The target domain consists of 500 fibers drawn from each bale and scanned by the longitudinal line scan system. Due to the varying lengths of the fibers, this generates approximately 65,000 to 125,000 tiles (and, hence, feature vectors) per bale. In order to keep the number of feature vectors the same among the categories, 65,000 data points were randomly sampled from each.

The first task is to determine which of the 14 line scan features are "good" features to use in the learning task. Initially, density estimates consisting of pairwise combinations of the 14 features were visually analyzed. For example, Fig. 4.1 shows 2D density estimates of shape factor and homogeneity for each of the five bales. The graphs have been ordered from the most mature, Bale 3112 in Fig. 4.1(a), to the least mature, Bale 3116 in Fig. 4.1(e) — a determination based on the distributions in Fig. 3.6. Both features have been independently normalized to zero mean and unit variance using a global sample mean and variance calculated from all samples among all bales. Note the gradual but distinct shift in the distribution of these features in both dimensions. This analysis is the motivation for the feature selection algorithm described Chapter II. An exhaustive visual examination of all pairwise combinations reveals that all of the features based on fiber width along with the texture-based features homogeneity and contrast seem to be potentially good features. Interestingly, none of the intensity-based features appeared to show any promise. Also, Haralick features energy and coarseness seemed to provide no useful information as the class conditional densities for these features, $P\left(x^{T} \mid c_{j}^{T}\right)$, were almost identical. 
Texas Tech University, Christopher N. Turner, December 2016

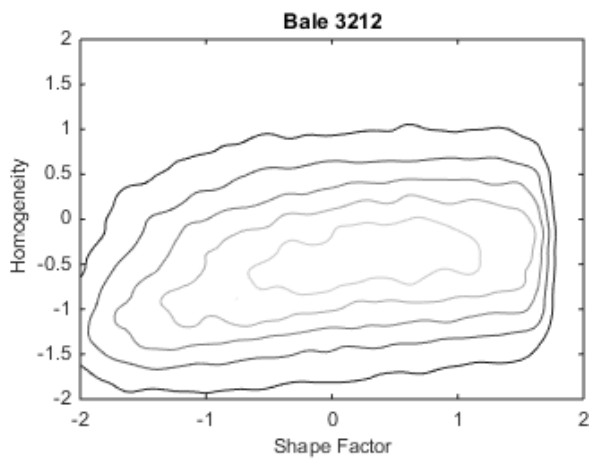

(a)

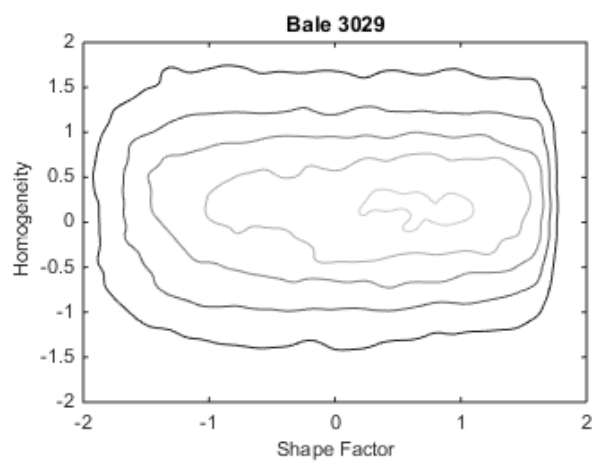

(c)

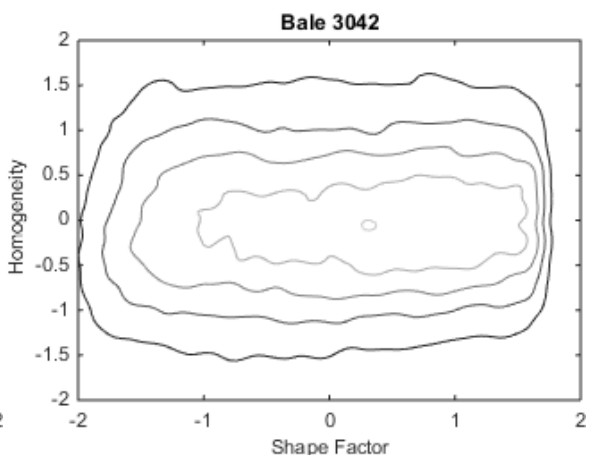

(b)

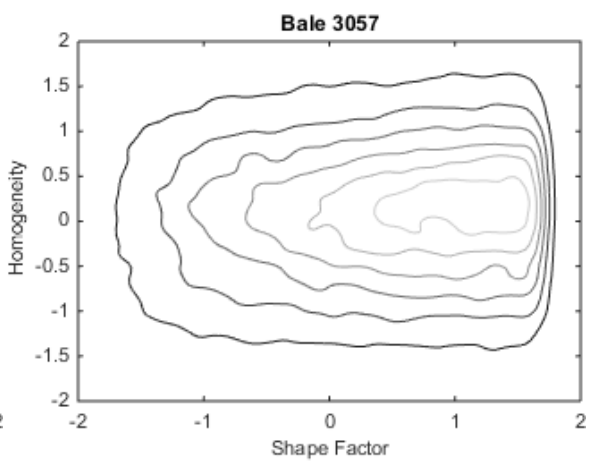

(d)

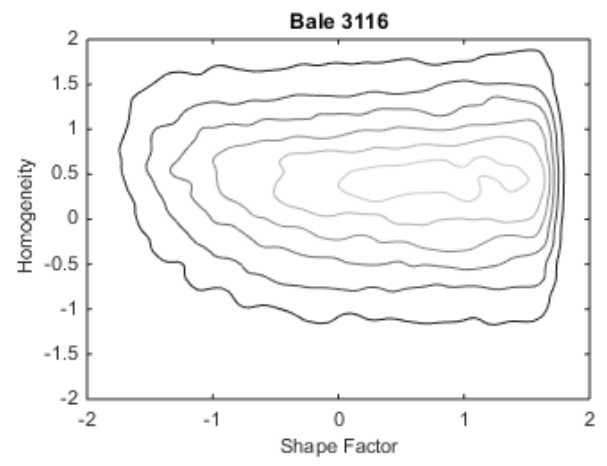

(e)

Fig. 4.1. Contour plots of density estimates of samples from the target domain for two particular features, i.e. $P\left(x^{T} \mid c_{j}^{T}\right)$ : shape factor and homogeneity. Graphs are arranged from highest maturity to lowest maturity according to the distributions in Fig. 3.6: (a) Bale 3212, (b) Bale 3042, (c) Bale 3029, (d) Bale 3057, and (e) Bale 3116.

Applying the proposed feature selection algorithm on this data set produces results that generally correspond to the observations made while examining 2D density 
estimates. Bale 3212, the most mature cotton, was used as the reference category. Table 4.1 shows the ordered list of features removed by the algorithm in reverse order. In other words, coarseness was the first feature to be removed, and homogeneity was the last remaining feature. Fig. 4.2(a) shows the value of the criterion function as each feature was removed.

Table 4.1 Ranked List of Features*

\begin{tabular}{ll}
\hline 1. Homogeneity & 8. Fiber Width Avg \\
2. Contrast & 9. Correlation \\
3. Shape Factor & 10. Intensity Max \\
4. Fiber Width Std Dev & 11. Intensity Min \\
5. Fiber Width Max & 12. Intensity Avg \\
6. Fiber Width Min & 13. Energy \\
7. Intensity Std Dev & 14. Coarseness \\
\hline
\end{tabular}

*Ranked list of features removed from the algorithm in reverse order. Coarseness was the first feature removed by the algorithm, and homogeneity was the last.

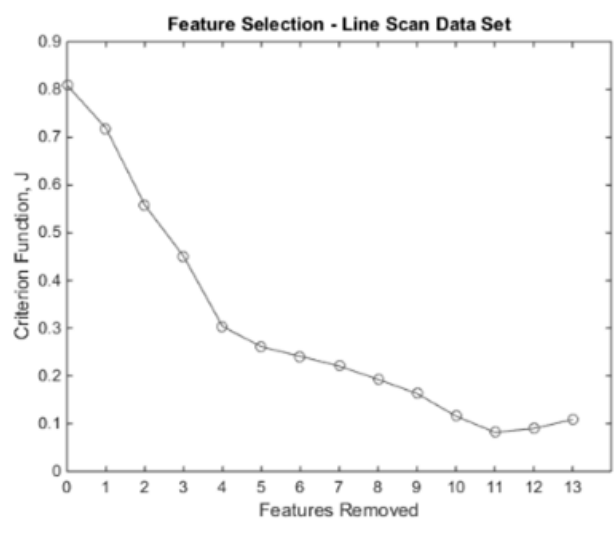

(a)

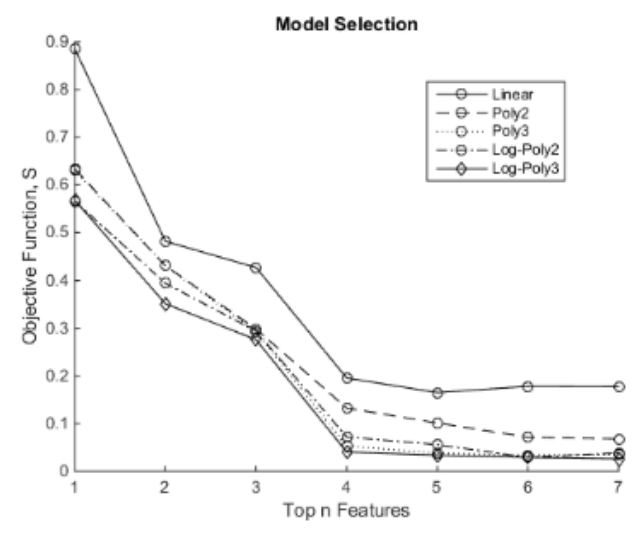

(b)

Fig. 4.2. (a) Results of running the feature selection algorithm on the line scan data set. Each point shows the value of the criterion function as each feature is removed. The minimum occurs when the eleventh feature is removed. (b) Results of applying five different models to subsets of the top seven features.

Using the top five features from Table 4.1, we applied the modeling algorithm to four different models: linear, quadratic, logistic with a linear exponential term, and 
logistic with a quadratic exponential term. Table 4.2 shows the results for each model. Clearly, the logistic model with the quadratic exponent showed the best results indicated by the lowest value of $M(0.037)$ and the highest $R^{2}(0.96)$. It should be noted that these results are better than the performance of the best model in the previous example (Table 3.5). In that example, Fig. 3.8(b) shows evidence that the model provides a very good approximation in the estimation of maturity, and the model performance measures reflect that. In this case, we can deduce that if true maturity values were available, then based on the model performance measures $M$ and $R^{2}$, we would see a very similar graph to that of Fig. 3.8(b) - if not better. Fig. 4.3 compares the resulting label distributions for each bale used in training between the source and target domains. At the top of each graph in Figs. 4.3(a)-(e), we provide the Jensen-Shannon Divergence (JSD) [37] as a quantitative measure of similarity between the source and target label distributions.

Table 4.2 Model Selection - Vision-Based Maturity measurement

\begin{tabular}{lcc}
\hline Model Type & $M$ (Eq. (2.9)) & $R^{2}$ (Eq. (2.10)) \\
\hline Linear & 0.055 & 0.91 \\
Quadratic & 0.044 & 0.94 \\
Logistic-Linear & 0.048 & 0.93 \\
Logistic-Quadratic & $\mathbf{0 . 0 3 7}$ & $\mathbf{0 . 9 6}$ \\
\hline
\end{tabular}




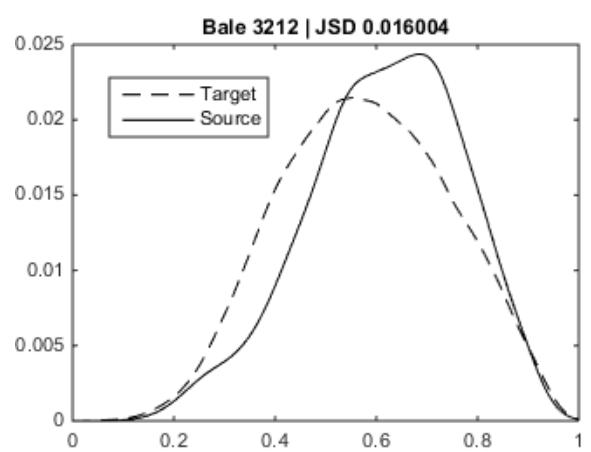

(a)

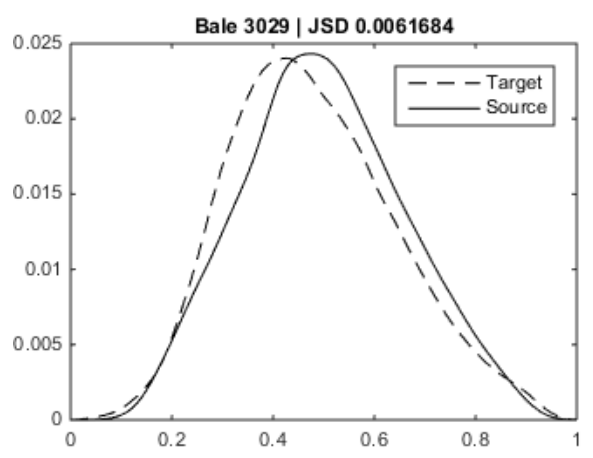

(c)

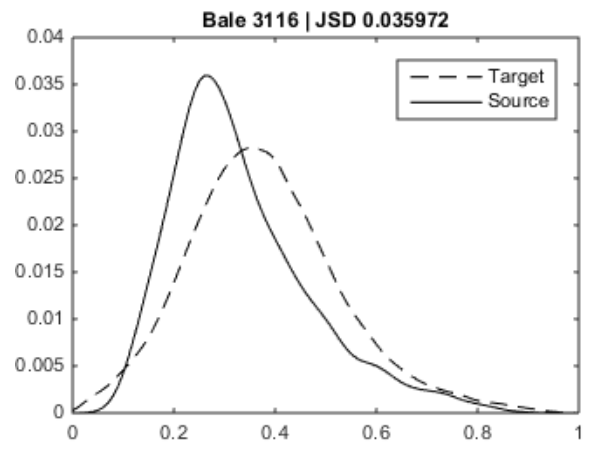

(e)

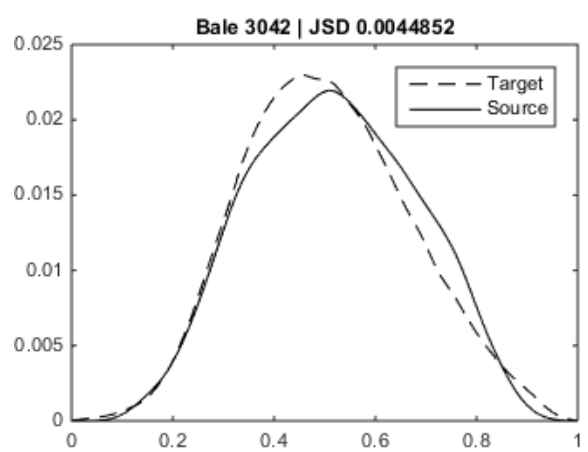

(b)

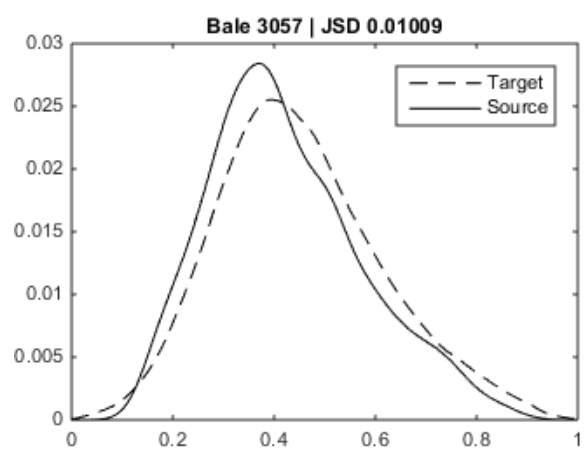

(d)

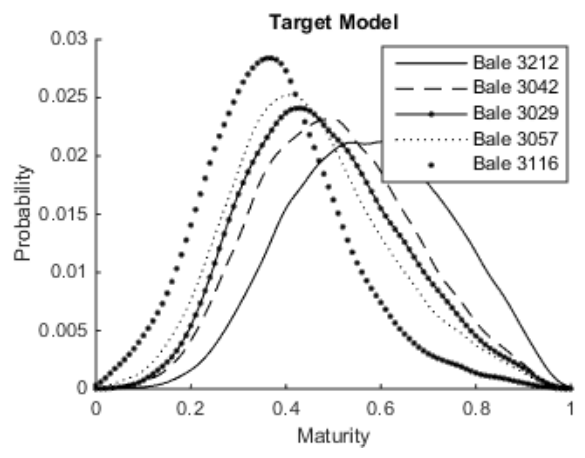

(f)

Fig. 4.3. (a)-(e) Density estimate of maturity for each of the five cotton bales in the cross section domain (source) and the predicted maturity based on the "LogisticQuadratic" model for the vision-based measurement (target). (f) Distribution of predicted maturity for each bale in the target domain. Compare to Fig. 3.6. 
For validation of the chosen model, we have employed a hold-out method in which samples from three additional bales not used in training were tested. Fig. 4.4 shows the source label distributions as well as the predicted target label distributions of the samples taken from each of the test bales. Bale 3129 (Fig. 4.4(a)) is a more immature type of cotton, similar to Bale 3116 (Fig. 4.3(e)), which was used in training. In each of these graphs, the manner in which the source and target distributions diverge is similar-i.e. both target label distributions indicate a higher density of slightly more mature fibers than the source. We believe this may be due to the fact that immature fibers are more difficult to handle individually. They are often twisted and relatively brittle. Because the prototype system requires a human to manually place fibers on slides to be imaged, we posit that many of the more immature fibers were broken (resulting in a smaller length and, therefore, fewer image tiles) or simply discarded. However, Bale 3056 (Fig. 4.4(b)) along with other "middle maturity" cottons used in training, Bales 3042, 3029, and 3057 (Figs. 4.3(b)-(d), respectively), exhibit very similar source and target label distributions - as is indicated by the lower JSD values. Also, the more mature test sample from Bale 3140 (Fig. 4.4(c)) also shows a strong resemblance between source and target domains. It should be noted that the JSD for this particular result (0.011) appears to be slightly better than the results of the mature cotton used in training, Bale 3212 (Fig. 4.3(a)) with a JSD of 0.016. We believe this illustrates that the chosen model generalizes quite well. 


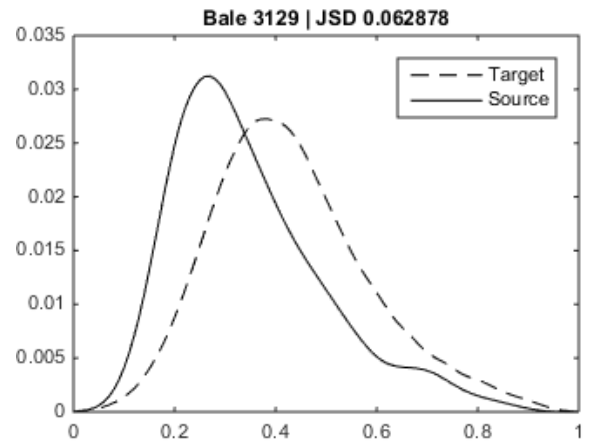

(a)

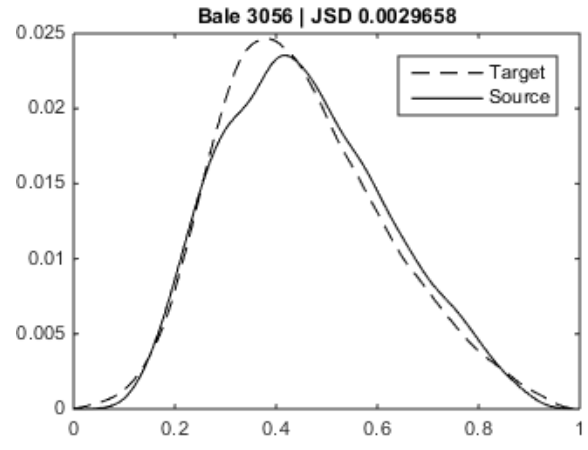

(b)

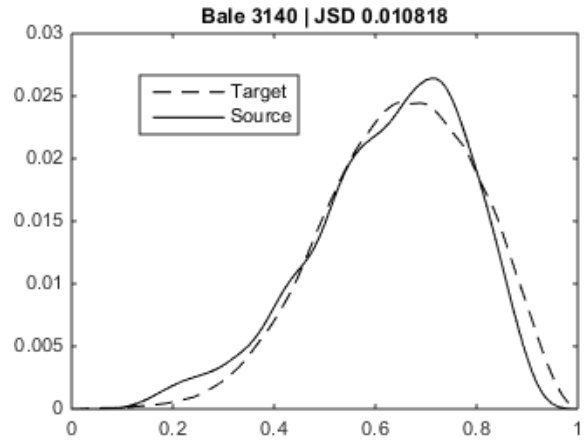

(c)

Fig. 4.4. Results of samples taken from three bales used to test the chosen "LogisticQuadratic" model. Each graph shows the source label distributions as well as the predicted target label distributions. (a) Bale 3129 (b) Bale 3056 (c) Bale 3140

As an additional validation step, we visually examined fiber segments in relation to their predicted maturity values. To the experienced researcher, subtle differences in cotton maturity can be observed in longitudinal views of cotton fibers. However, armed with a basic knowledge of cotton maturity (as explained above), extreme differences in maturity are readily apparent to most individuals. To that end, using the best model indicated in Table 4.2, Fig. 4.5 presents 12 of the most mature and 12 of the most immature fiber segments for Bale 3056 (one of the test bales, shown in Fig. 4.4(b)) along with their predicted maturity values. Mature fibers are full of cellulose and appear full and round, while immature fibers have almost no cellulose and become an empty tube often exhibiting a flat, ribbon-like appearance. The fiber segments in both figures exhibit these qualities adding further validation to the results. 
Furthermore, experts in cotton research have examined these results as well as many other images and have affirmed that the results are very reasonable.

(a)

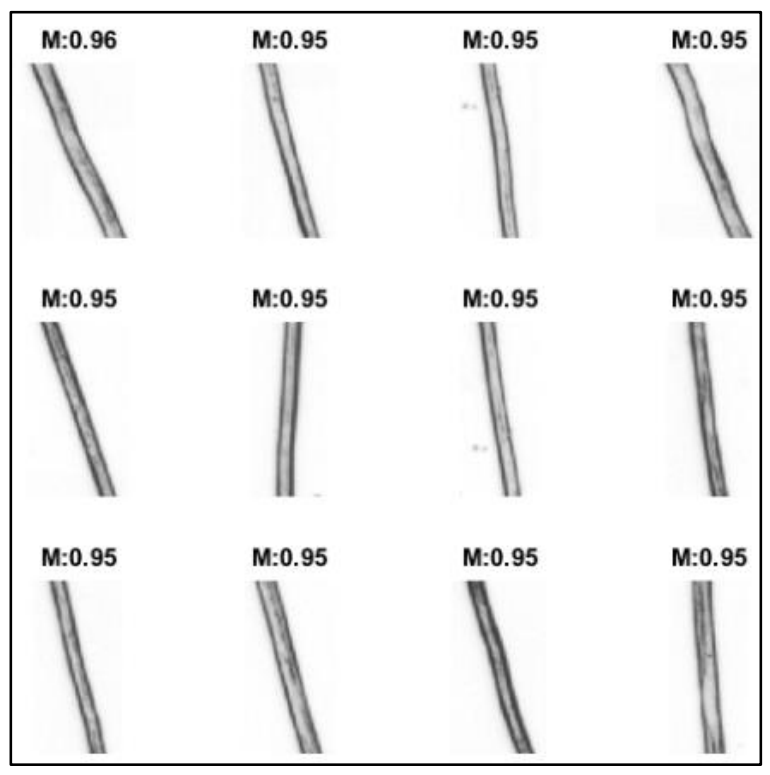

(b)

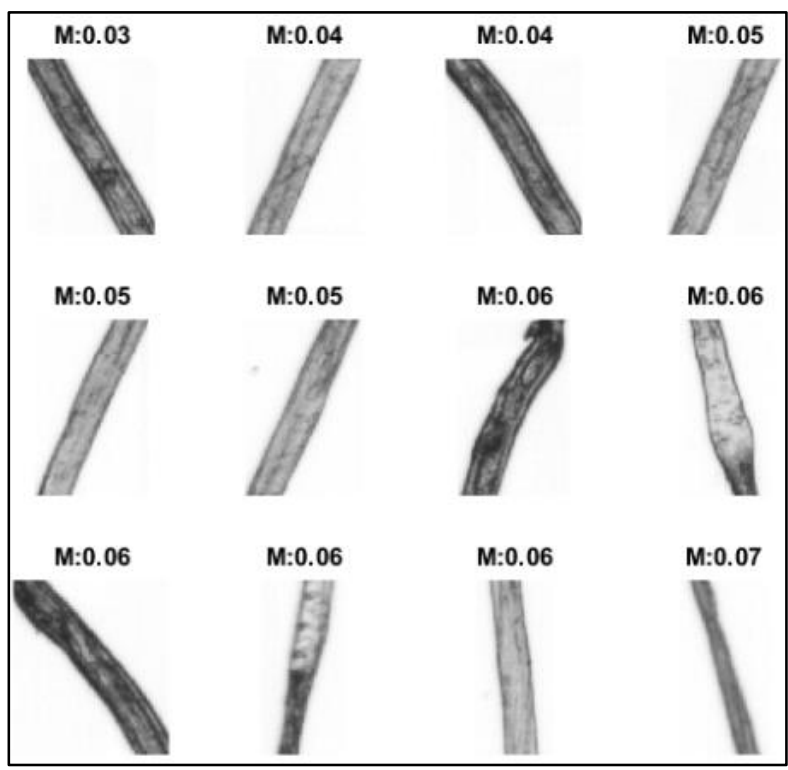

Fig. 4.5. Twelve of the most (a) mature and (b) immature fiber segments from Bale 3056 using predicted values based on the "Logistic-Quadratic" model. Predicted maturity values appear above each segment. 
Texas Tech University, Christopher N. Turner, December 2016

\section{CHAPTER V CONCLUSIONS}

This dissertation presents novel feature selection and modeling algorithms for a transductive transfer learning regression setting. Both algorithms make use of a single feature common to both the source and target domains to create a link with which to transfer information regarding distributions of data in the feature space as well as the label space. The novelty of the presented solution stems from the fact that no other work has presented a transfer regression algorithm in which there are no labeled data in the target domain and the dimensionality of the two domains is different. The synthetic examples for the feature selection algorithm indicate that it is very robust when there is a large degree of overlap among the categories - a scenario which is very likely in practice. Then for the modeling algorithm, the first example uses synthetic data and offers a simple, visual illustration of how the algorithm works in a nearly ideal case. In the second example, we show that the algorithm supports different regression models and methods and is not dependent on any relationship in the distribution of features between the two domains-i.e. there is no assumed relationship between $P\left(x^{S} \mid c_{j}^{S}\right)$ and $P\left(x^{T} \mid c_{j}^{T}\right)$. The third example illustrates the effectiveness of the algorithm in a realistic scenario where one instrument's data (Cottonscope) is utilized to learn how to predict the same measurement using a completely different measurement method (cross section method). The results show that the learned model is a close approximation to the true model in evaluating maturity. Finally, we apply the algorithm to a new vision-based measurement system for evaluating cotton fiber maturity. The model performance measures along with the results from three test cottons indicate that the resulting model enables the new visionbased instrument to assess maturity quite well. This result is further validated by cotton research experts' visual assessments. 


\section{BIBLIOGRAPHY}

[1] D. Schneider, Y.S. Gloy, and D. Merhof, "Vision-based on-loom measurement of yarn densities in woven fabrics," IEEE Trans. Instrum. Meas., vol. 64, no. 4, pp. 1063-1074, 2015.

[2] A. Song, Y. Han, H. Hu, et al., "A novel texture sensor for fabric texture measurement and classification," IEEE Trans. Instrum. Meas., vol. 63, no. 7, pp. 1739-1747, 2014.

[3] S. Matteoli, M. Diani, R. Massai, et al., "A spectroscopy-based approach for automated nondestructive maturity grading of peach fruits," IEEE Sens. J., vol. 15, no. 10, pp. 5455-5464, 2015.

[4] A. Vermuelen, L. Verdonck, and J.M. Kaufman, "A critical evaluation of simple methods for the estimation of free testosterone in serum," J. Clin. Endocrinol. Metab., vol. 84, no. 10, pp. 3666-3672, 1999.

[5] B. Kelly, N. Abidi, D. Ethridge, and E. Hequet, "Fiber to Fabric," In D. Fang and R. Percy (Ed.), Cotton (Second edition), American Society of Agronomy, 2015, pp. 665-744.

[6] AATCC, Test method 20A-2012, AATCC Technical Manual, 2012.

[7] J. Bradow, O. Hinojosa, L. Wartelle, et al., "Applications of AFIS fineness and maturity module and X-ray fluorescence spectroscopy in fiber maturity evaluation," Text. Res. J., vol. 66, pp. 545-554. 1996.

[8] S. Gordon, G. Naylor, M. Brims, et al., "Cottonscope: a new instrument for the maturity and fineness measurements, (a) Instrument design," In Proc. $31^{\text {st }}$ Int. Cotton Conf, Bremen, Germany, March 2012, pp. 127-136.

[9] J. Rodgers and D. Thibodeaux, "Cottonscope: A new instrument for the maturity and fineness measurements, (b) Experimental results and experiences," In Proc. $31^{\text {st }}$ Int. Cotton Conf., Bremen, Germany, March 2012, pp. 143-153.

[10] M. Shahriar, I. Scott-Fleming, H. Sari-Sarraf, and E. Hequet, "A machine vision system to estimate cotton fiber maturity from longitudinal view using a transfer learning approach," Mach. Vision Appl., vol. 24, no. 8, pp. 1661-1683, May 2013.

[11] C. Turner, H. Sari-Sarraf, E. Hequet, and S. Vitha, "Variation in maturity observed along individual cotton fibers using confocal microscopy and image analysis,” Text. Res. J., vol. 85, no. 8, pp. 867-883, May 2015. 
[12] E. Hequet, B. Wyatt, N. Abidi, et al., "Creation of a set of reference material for cotton fiber maturity measurements," Text. Res. J., vol. 76, pp. 576-586, 2006.

[13] S. J. Pan and Q. Yang, "A survey on transfer learning," IEEE Trans. Knowl. Data Eng., vol. 22, no. 10, pp. 1345-1359, Oct. 2010.

[14] M. Long, J. Wang, G. Ding, S. J. Pan, and P. S. Yu, “Adaptation regularization: A general framework for transfer learning," IEEE Trans. Knowl. Data Eng., vol. 26, no. 5, pp. 1076-1089, May 2014.

[15] L. Zhang and D. Zhang, "Domain adaptation extreme learning machines for drift compensation in E-nose systems," IEEE Trans. Instrum. Meas., vol. 65, no. 7 , pp. 1790-1801, 2015.

[16] B. Kulis, K. Saenko, T. Darrell, "What you saw is not what you get: Domain adaptation using asymmetric kernel transforms," in Proc. of CVPR, 2011, pp. 1785-1792.

[17] K. Saenko, B. Kulis, M. Fritz, and T. Darrell, “Adapting visual category models to new domains," in Proc. ECCV 2010, pp 213-226.

[18] H. Bay, A. Ess, T. Tuytelaars, and L. Van Gool, "Surf: Speeded up robust features," Comput. Vis. Image Und., vol. 110, no. 3, pp. 346-359, 2008.

[19] D. G. Lowe, "Distinctive image features from scale-invariant keypoints," Int. J. Comp. Vis., vol. 60, no. 2, pp. 91-110, Nov. 2004.

[20] W. Dai, Y. Chen, G. Xue, Q. Yang, and Y. Yu, “Translated learning: Transfer learning across different feature spaces," In NIPS, pages 353-360, 2008.

[21] B. Tan, E. Zhong, M. Ng, and Q. Yang, "Mixed-transfer: Transfer learning over mixed graphs," in Proc. of SIAM Int. Conf. on Data Mining, 2014, pp. 208-216.

[22] P. Yang, Q. Tan, and Y. Ding, "Bayesian task-level transfer learning for nonlinear regression," in Proc. Int. Conf. Comput. Sci. Software Eng., 2008, pp. 62-65.

[23] W. Mao, G. Yan, J. Bai, and H. Li, "Regression transfer learning based on principal curve," in Lecture Notes on Computer Science, vol. 6063. Berlin Heidelberg: Springer, 2010, pp. 365-372. 
[24] J. Liu, Y. Chen, and Y. Zhang, "Transfer regression model for indoor 3-D location estimation," in Lecture Note on Computer Science, vol. 5916. Berlin Heidelberg: Springer, 2010, pp. 603-613.

[25] D. Pardoe and P. Stone, "Boosting for regression transfer," in Proc. Int. Conf. Mach. Learning, 2010, pp. 863-870.

[26] Z. Deng, K. Choi, Y. Jiang, and S. Wang, "Generalized hidden-mapping ridge regression knowledge-leveraged inductive transfer learning for neural networks, fuzzy systems and kernel methods," IEEE Trans. Cybern., vol. 44, no. $12,2014$.

[27] S. J. Pan, J. T. Kwok, and Q. Yang, "Transfer learning via dimensionality reduction," in Proc. 22nd AAAI, 2008, pp. 677-682.

[28] T. Helleputte and P. Dupont, "Feature selection by transfer learning with linear regularized models," in Lecture Notes in Artificial Intelligence, vol. 5781, pp. 533-547, 2009.

[29] S. Uguroglu and J. Carbonell, "Feature selection for transfer learning," in Lecture notes in Computer Science, vol. 6913, 2011, pp. 430-442.

[30] J. Gao, "A local domain adaptation feature extraction method," in 10th Int. Conf. FSKD, 2013, pp. 526-530.

[31] K. M. Borgwardt, A. Gretton, M. J. Rasch, H.-P. Kriegel, B. Schölkopf, and A. J. Smola, "Integrating structured biological data by kernel maximum mean discrepancy," Bioinformatics, vol. 22, no. 14, pp. e49-e57, 2006.

[32] A. Gretton, K. M. Borgwardt, J. Rasch, B. Schölkopf, and A. Smola, "A kernel two-sample test," J. of Mach. Learn. Res., vol. 13, pp. 723-773, Mar. 2012.

[33] T. Marill and D.M. Green, "On the effectiveness of receptors in recognition system," IEEE Trans. Inf. Theory, vol. 9, no. 1, pp. 11-17, Jan. 1963.

[34] R. C. Gonzalez and R. Woods, Digital Image Processing, Addison-Wesley Publishing Company, 1992, pp. 173-182.

[35] S. Theodoridis and K. Koutroumbas, Pattern Recognition (Fourth edition), Academic Press, 2009, pp. 49-56.

[36] R. M. Haralick, "Statistical and structural approaches to texture," Proc. of the IEEE, vol. 67, no. 5, pp. 786-804, May 1979.

[37] D. M. Endres and J. E. Schindelin, "A new metric for probability distributions," IEEE Trans. Info. Theory, vol. 49, no. 3, pp. 1858-1860, 2003. 